\title{
Les relations gréco-turques au tournant du siècle : ruptures, évolutions et permanences
}

Greek-Turkish relations at the turn of the century: Ruptures, evolutions and permanencies

Yüzyılın başında Türk-Yunan ilişkiler: tatili, değişiklikler, hatları

\section{Samim Akgönül}

\section{(2) OpenEdition}

\section{Journals}

Édition électronique

URL : https://journals.openedition.org/ceb/4613

DOI : $10.4000 /$ ceb.4613

ISSN : 2261-4184

Éditeur

INALCO

Édition imprimée

Date de publication : 1 janvier 2004

ISSN : 0290-7402

Référence électronique

Samim Akgönül, « Les relations gréco-turques au tournant du siècle : ruptures, évolutions et

permanences », Cahiers balkaniques [En ligne], 33 | 2004, mis en ligne le 04 juin 2013, consulté le 06 juillet 2021. URL : http://journals.openedition.org/ceb/4613 ; DOI : https://doi.org/10.4000/ceb.4613

Ce document a été généré automatiquement le 6 juillet 2021.

Cahiers balkaniques est mis à disposition selon les termes de la Licence Creative Commons Attribution - Pas d'Utilisation Commerciale 4.0 International. 


\title{
Les relations gréco-turques au tournant du siècle : ruptures, évolutions et permanences
}

\author{
Greek-Turkish relations at the turn of the century: Ruptures, evolutions and \\ permanencies \\ Yüzyılın başında Türk-Yunan ilişkiler: tatili, değişiklikler, hatları
}

Samim Akgönül

\section{Introduction}

1 Le terme de "relations gréco-turques " peut être compris de plusieurs manières. La signification la plus répandue, utilisée aussi bien dans les études académiques qu'en langage courant, concerne les relations bilatérales qu'entretiennent les États grec et turc. Mais de nouvelles dimensions doivent impérativement être ajoutées à cette grille d'analyse, compte tenu de l'internationalisation des conflits bilatéraux, du développement des démocraties grecque et turque et enfin de l'explosion spectaculaire des moyens de communication. En effet, dans la dernière décennie du $\mathrm{xx}^{\mathrm{e}}$ siècle, les conflits bilatéraux proprement dits ont pratiquement disparu de l'échiquier international, du moins dans le monde occidental. Étant donné que la Grèce et la Turquie appartiennent à ce monde de complexification du jeu international, il est normal que leurs relations sortent du cadre strict d'un conflit d'intérêts entre deux États. Les autres angles d'analyse, à savoir les relations entre la nation turque et la nation grecque, entre les individus, entre les différentes formes de la société civile sont à prendre en considération, bien que pour l'instant ils ne prennent pas le pas sur les relations étatiques proprement dites.

2 À la multiplication des angles d'analyse et des acteurs, il faut ajouter la multiplication et complexification des composantes du conflit également. En effet, au fur et à mesure que les différends gréco-turcs se sclérosaient, de nouveaux terrains de compétition se 
sont ajoutés au conflit bilatéral. D'une manière classique, à la fin du $\mathrm{xx}^{\mathrm{e}}$ siècle, on peut énumérer les composantes de ce conflit comme suit :

1. Chypre.

2. Mer Égée :

a) Plateau continental (seul problème aux yeux de la Grèce),

b) Eaux territoriales,

c) Flight Information Region (FIR),

d) Démilitarisation/Militarisation des îles égéennes,

e) « Zones grises ».

3. Minorités :

a) Minorité musulmane de Thrace occidentale (Question de la reconnaissance identitaire, problèmes des muftis, problèmes scolaires),

b) Minorité grecque orthodoxe de Turquie (Patriarcat, École théologique de Halki, fondations pieuses, vieillissement de la population, écoles minoritaires).

4. Question kurde en Turquie (Affaire Öcalan, camp de réfugiés de Lavrion),

5. Relations turco-européennes.

3 Pendant ces dix dernières années, les points de friction et de tensions suscités ont connu des destins divers. On a pu observer des ruptures avec changements de situation radicaux, des évolutions lentes, mais sensibles, mais aussi des continuités dans certains domaines. Il est bien entendu impossible de traiter d'une manière totalement indépendante les différends en question tant ils sont imbriqués. Ainsi, pour plus de clarté de lecture, je mentionnerai d'abord les points de rupture qui ont fait que l'attitude des deux États a totalement changé vis-à-vis de certains problèmes; ensuite j'analyserai les domaines où il y a eu des évolutions sensibles en partie liées aux ruptures mentionnées et enfin j'évoquerai quelques points de stagnation dus en grande partie à l'attitude de certains milieux. Il est également difficile de prendre en considération comme un tout les politiques grecques à l'égard de la Turquie, ou les politiques turques à l'égard de la Grèce. Dans les deux pays, les acteurs agissant sur les relations bilatérales étant de plus en plus divers, les politiques étatiques ne sont plus les seules déterminantes.

\section{Points de rupture}

\section{Le nouvel ordre mondial : la redistribution des cartes régionales}

Les relations gréco-turques n'évoluent pas en circuit fermé. Ainsi, une des raisons les plus importantes de rupture dans les politiques bilatérales de la décennie 1990 doit être cherchée dans les bouleversements de la carte géopolitique du monde après l'effondrement des régimes communistes en Union soviétique et dans l'Europe de l'Est. Dans le cadre macropolitique, la place de la Turquie et de la Grèce dans le nouvel ordre mondial a été totalement à redéfinir. C'est durant cette décennie que les responsables turcs ont eu peur de perdre leur place stratégique aux yeux de la seule superpuissance américaine, tandis que les dirigeants grecs tentaient d'en découdre avec les vieux démons du nationalisme pour devenir une démocratie occidentale européenne. Les premiers ont été rassurés : avec la première guerre du Golfe, les alliés/tuteurs/ protecteurs américains ont compris que la Turquie était un pays dont la situation géopolitique restait hautement stratégique. Quant aux seconds, c'est seulement dans la 
deuxième moitié des années 1990 qu'ils placèrent la Grèce en Europe, économiquement et politiquement parlant. C'est dans ces circonstances nouvelles qu'il leur fallait aborder les relations bilatérales.

Les changements depuis la chute des régimes communistes ne sont pas limités à la conjoncture globale. Au niveau régional également il y a eu des modifications radicales qui ont obligé les dirigeants grecs et turcs à se repositionner. En effet, dès le début des années 1990, la Turquie renoue avec les Balkans et établit des relations cordiales, parfois étroites, avec la Bulgarie, la Bosnie ou la Macédoine. Cette situation sans précédent a accentué le sentiment d'encerclement déjà présent dans l'opinion publique grecque. Cela dit, dans les tout premiers mois qui ont suivi l'effondrement du bloc communiste, un vent d'optimisme a soufflé en Grèce. Avec la fin de la guerre froide, l'importance stratégique de la Turquie pourrait diminuer, pensait-on, et enfin un équilibre gréco-turc serait établi aux yeux de Washington ${ }^{1}$. Mais la guerre du Golfe, suivie des problèmes avec les pays balkaniques (Albanie et Macédoine principalement), mais aussi de la guerre de Yougoslavie où la Grèce s'est trouvée isolée, enfermée qu'elle était dans une solidarité religieuse avec les Serbes, ont fait que ce vent d'optimisme a vite laissé place à un repli identitaire et à une exaspération nationaliste. Certes, le danger ne venait plus du Nord, mais de l'Est, mais le Nord était en affinité avec l'Est. D'autant plus que ce point de rupture dans les relations gréco-turques qu'est la fin de la guerre froide fut accompagné par une nouvelle compétition entre les deux États dans leur quête pour devenir une puissance régionale. C'est ainsi qu'un nouveau domaine de rivalité est apparu dans l'axe Turquie/Balkans/Asie Centrale et Grèce/Balkans/Europe, sans compter les nouvelles alliances au Proche-Orient (Turquie-Israël, Grèce-Syrie).

\section{Bataille pour un rocher : exaspération populiste}

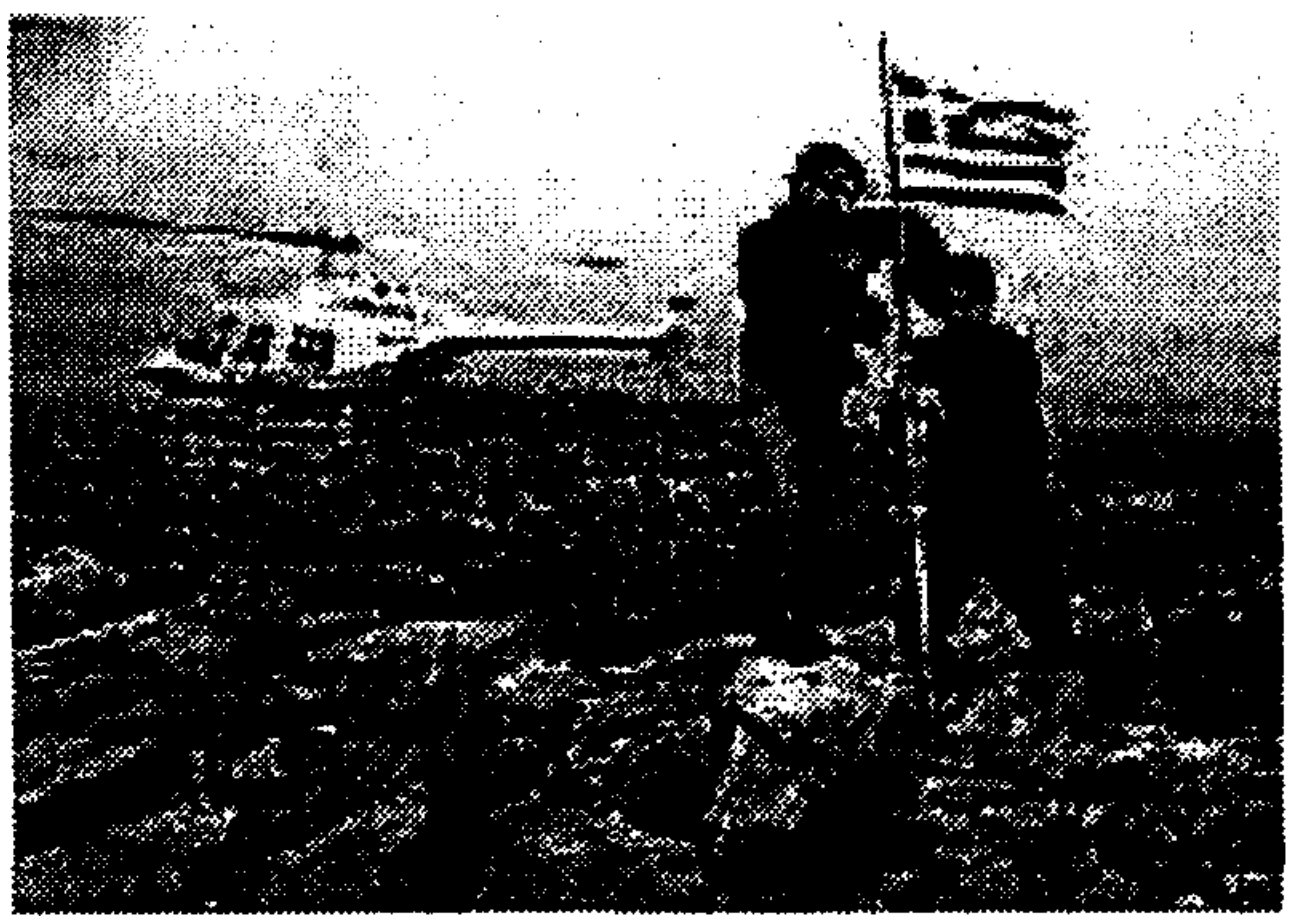


6 Ce point de rupture global est suivi par d'autres, régionaux, bilatéraux ou conjoncturels. Concernant un des aspects les plus techniques du conflit gréco-turc, celui de la mer Égée, le point de rupture a eu lieu en janvier 1996. Le 25 décembre 1995, un cargo turc a échoué au large d'un groupe de rochers près de la petite ville turque balnéaire de Turgutreis. Ces rochers ont été dotés immédiatement de deux noms: Kardak pour les Turcs, et Imia pour les Grecs. Les autorités grecques qui ont porté secours à ce cargo ont été accusées par les autorités turques d'avoir violé les eaux territoriales turques. Après un échange de notes diplomatiques plus ou moins violentes, ce sont les forces populistes et nationalistes des deux pays qui sont entrées en jeu et une course au drapeau a commencé. Le 26 janvier, le maire de l'île de Kalymnos est venu sur les rochers pour y planter le drapeau grec. Immédiatement après, deux « journalistes » du quotidien populiste turc Hürriyet arrivés en hélicoptère sur cet îlot y ont planté un drapeau turc. La marine grecque est intervenue pour enlever ce drapeau et le remplacer par un drapeau grec pour affirmer la souveraineté grecque sur ces rochers. En réponse, l'armée turque a envoyé un commando sur un autre rocher à proximité. La presse des deux pays a outrageusement exploité l'événement en évoquant la possibilité d'une "guerre des Falklands » entre la Grèce et la Turquie ${ }^{2}$. Il se trouve qu'au même moment, la Turquie se trouvait en plein milieu d'une crise politique où Tansu Çiller, alors premier ministre, était confrontée à la difficulté de former un nouveau gouvernement. De manière provocatrice, elle s'est emparée de cet incident pour créer une exaspération nationaliste encouragée par la presse ${ }^{3}$. En Grèce non plus, la situation politique n'était pas stable. Après le retrait d'Andréas Papandréou, après des luttes internes, l'aile modérée du PASOK s'était emparée de la direction du parti et avait formé son premier gouvernement sous la direction de Costas Simitis. Lorsque la crise d'Imia/Kardak a éclaté, le gouvernement Simitis, avant même d'avoir obtenu le vote de confiance de la Vouli, s'est trouvé prisonnier des attitudes nationalistes habituelles du PASOK, car l'aile dure de de son parti était toujours présente en la personne de Théodoros Pangalos au ministère des Affaires étrangères. L'escalade s'est arrêtée là, grâce à l'intervention du Président américain Clinton le 31 janvier ${ }^{4}$. Il était clair que les deux pays avaient connu une grosse frayeur et cet événement montrait la fragilité du statu quo ${ }^{5}$. Alors qu'en raison de ce point de rupture, les relations bilatérales sont entrées dans une nouvelle phase de détente, à l'instar - toutes proportions gardées - de la Détente qui a suivi la crise de Cuba, un nouvel élément de conflit fait son apparition, celui des « zones grises». En effet, depuis 1996, les autorités turques soutiennent l'idée que certaines zones de la mer Égée n'ont pas un statut clair et veulent inclure ce problème dans le conflit plus général de la mer Égée ${ }^{6}$. Pour la Grèce, il n'y a qu'un seul conflit égéen, celui concernant le plateau continental et son règlement doit être confié à La Haye ${ }^{7}$.

\section{Affaire Öcalan : l'ennemi de mon ennemi est mon ami}




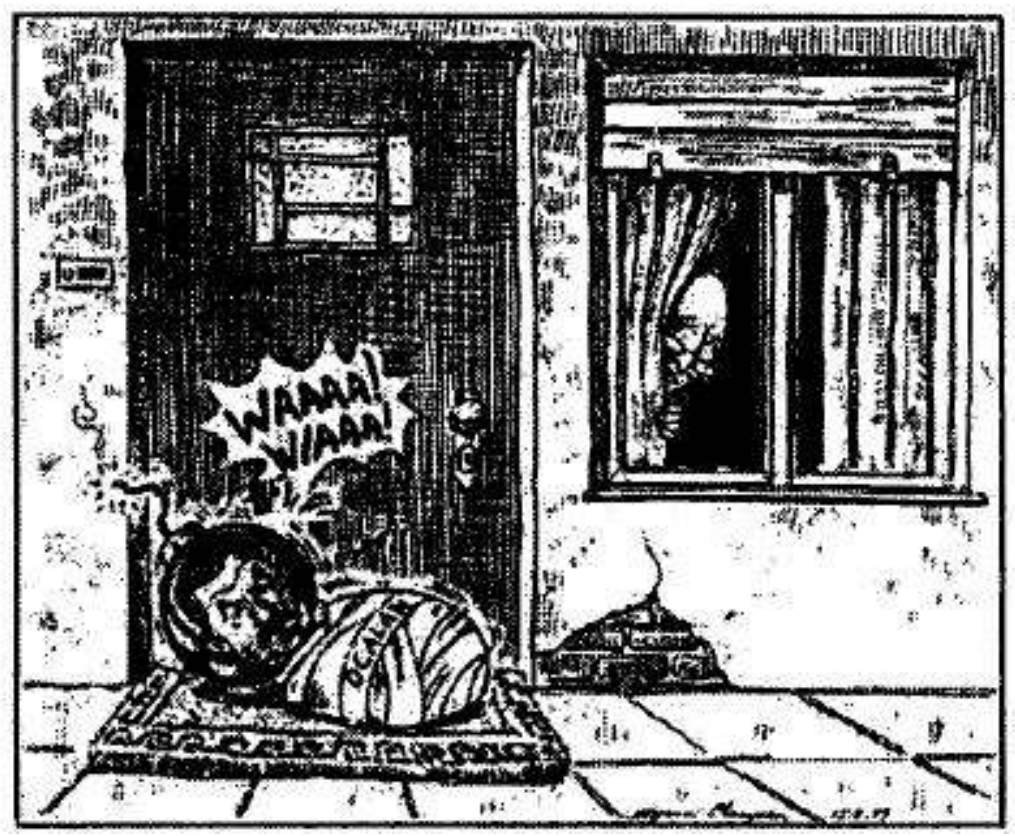

7 La crise d'Imia/Kardak est un vrai tournant vers l'amélioration des relations bilatérales. Les deux pays et les deux opinions publiques se sont soudain rendu compte qu'un affrontement armé n'était pas aussi improbable qu'on l'imaginait. Mais il va falloir attendre 1999 et l'affaire Öcalan pour voir une amélioration spectaculaire et générale des relations. Il faut tout de même préciser qu'Abdullah Öcalan, et d'une manière plus globale la question kurde, constitue, encore de nos jours, un des tabous les plus tenaces en Turquie. Depuis les années 1980 déjà, les autorités turques accusent la Grèce de vouloir profiter d'une des faiblesses de la Turquie en soutenant le $\mathrm{PKK}^{8}$ et son chef, et en entretenant un doute sur la fonction du camp de réfugiés de Lavrion. Au même titre que la Grèce craint les alliances entre la Turquie et les pays balkaniques tels que la Macédoine et l'Albanie, la Turquie craint également les alliances entre la Grèce et les pays comme la Syrie et l'Iran. L'affaire Öcalan a confirmé les inquiétudes de la Turquie en prouvant qu'effectivement certains milieux nationalistes grecs soutenaient la lutte armée du PKK et de son chef. En octobre 1998, devant la tension diplomatique entre la Syrie et la Turquie et les menaces de cette dernière d'attaquer la Syrie, Damas a été contraint d'extrader Abdullah Öcalan. Après avoir été refoulé par la Grèce dans un premier temps, il échoue à Moscou avant d'être arrêté à Rome le 12 novembre ${ }^{10}$. Après un bras de fer entre les autorités turques et italiennes (entre autres une campagne de boycott des produits italiens orchestrée par la presse turque ${ }^{11}$ ) le 16 janvier 1999, Abdullah Öcalan quitte le sol italien ${ }^{12}$ pour se rendre d'abord en Russie avant de venir en Grèce et de tenter une entrée aux Pays-Bas. C'est à ce moment-là que les milieux militaires et nationalistes grecs entrent en jeu. Face aux protestations de Costas Simitis qui craignait une détérioration de l'image de la Grèce, Öcalan fut transféré à Nairobi au Kenya ; après avoir refusé de loger chez un homme d'affaires grec, il s'est réfugié à l'ambassade grecque à Nairobi. Contraint de quitter l'ambassade, il a été récupéré par un commando turc pour être conduit en Turquie. 
« Deuxième "non" du gouvernement à Öcalan » (Ta Néa, 2 février 1999)

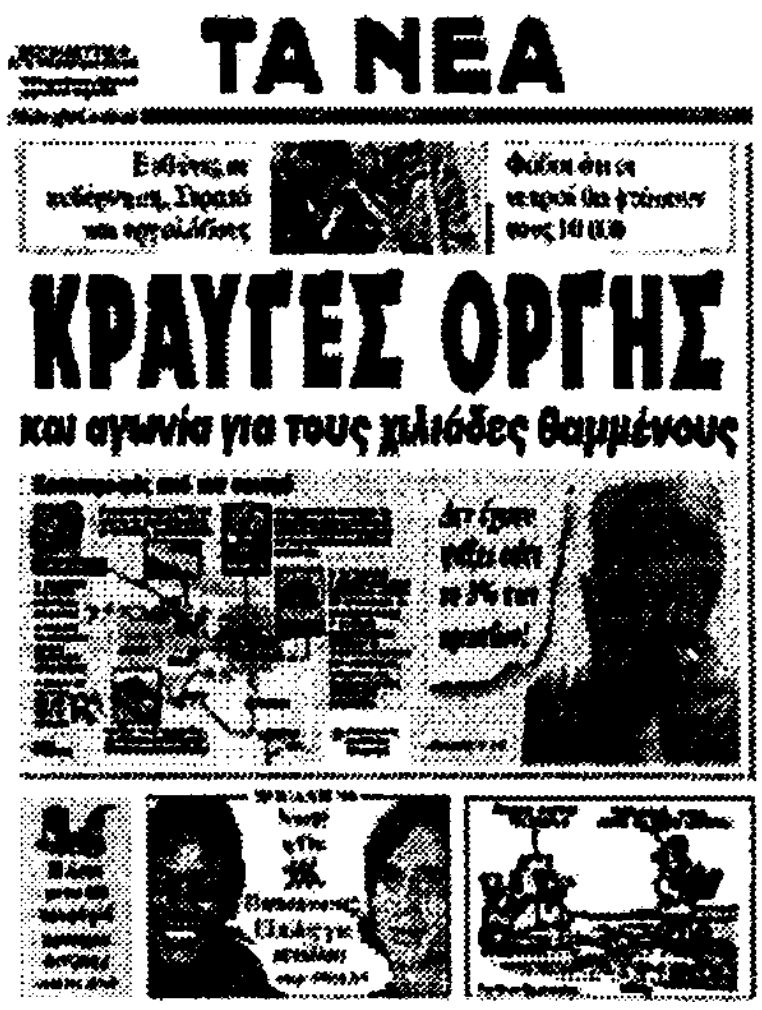

8 L'arrestation d'Öcalan est, pour la Turquie, un des événements les plus importants de la décennie, et l'implication de la Grèce a fourni beaucoup de raisons à Ankara pour accuser Athènes de soutenir le terrorisme. Le Président turc Süleyman Demirel a accusé la Grèce de soutenir le terrorisme et l'armée turque a été mobilisée. Il s'agissait de la même tactique d'intimidation utilisée contre la Syrie ${ }^{13}$.

Alors qu'on aurait pu s'attendre à une tension sans précédent entre les deux pays, pouvant déboucher sur un affrontement armé, l'arrestation d'Öcalan a, au contraire, ouvert une nouvelle phase de détente. L'attitude de Costas Simitis y est pour beaucoup. En effet, au lendemain de la crise, il a déclaré qu'il n'était pas au courant des événements et a limogé le ministre des Affaires étrangères Pangalos, le ministre de l'Intérieur Papadopoulos, et le ministre de l'Ordre public Petsalnikos ${ }^{14}$. On ne peut s'empêcher de penser que, grâce à la crise Öcalan, il a réussi à se débarrasser de la vieille garde nationaliste du PASOK et pût dorénavant montrer une attitude plus conciliante envers la Turquie. Une des conséquences principales de ce point de rupture fut l'arrivée au ministère des Affaires étrangères de Giorgos Papandréou, fils d'Andréas, mais toujours opposé aux politiques nationalistes et populistes de son père. Le tandem qu'ils ont formé avec le ministre des Affaires étrangères turc Ismail Cem a sans conteste contribué considérablement au redoux dans les relations bilatérales ${ }^{15}$. À partir de mars 1999, la politique grecque envers la Turquie a radicalement changé. Cette nouvelle détente allait culminer au sommet européen d'Helsinki en 1999 où, pour la première fois, la Grèce ne s'est pas mise en travers du processus d'intégration européenne de la Turquie.

\section{Séismes dans les relations entre Turcs et Grecs}




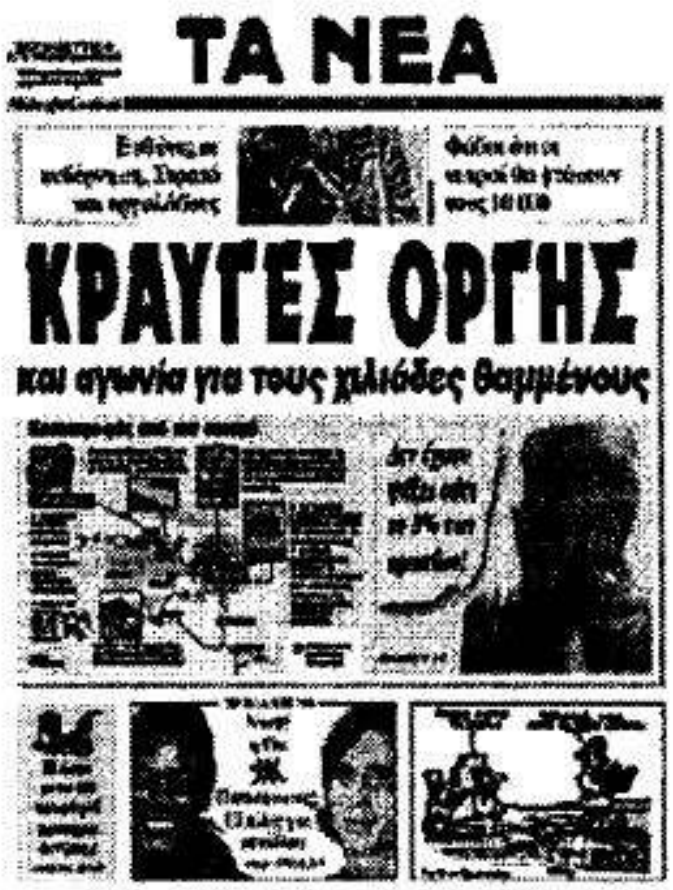

Ces changements au niveau politique ne seraient pas suffisants sans une rupture au niveau populaire. C'est en août 1999 que la Turquie fut frappée par un des séismes les plus violents de son histoire, plus précisément, le 17 août 1999 à 3 heures 7 du matin. Les images retransmises par les télévisions du monde entier dès les premières heures du tremblement de terre, les chiffres catastrophiques annoncés par les journalistes ${ }^{16}$ et l'impuissance relative des autorités turques face à cet événement sans précédent ont ému l'ensemble de la planète. La population de la Grèce n'est pas restée insensible non plus. Un élan de solidarité jamais vu apparaît dès la première journée. L'arrivée des secouristes grecs, les campagnes d'aide lancées par différents organismes en Grèce, une collecte de sang qui atteint des proportions inimaginables touchent de près l'opinion publique turque. Les journaux turcs qui jusque-là traitaient tout ce qui touche au Grec avec force préjugés et formules stéréotypées ont du jour au lendemain changé de ton. Plusieurs journaux ont titré des messages de remerciement en grec $^{17}$, les chaînes de télévision ont ouvert leurs journaux avec des messages d'amitiés en grec, ${ }^{18}$ etc. Mis à part quelques voix discordantes concernant l'acceptation des aides grecques (et arméniennes ${ }^{19}$ ) l'ensemble de la classe politique turque, les médias et les différentes organisations de la société civile ont manifesté leur gratitude au peuple (et à la classe politique ?) grec(s). 
Kathimerini, 19 août 1999

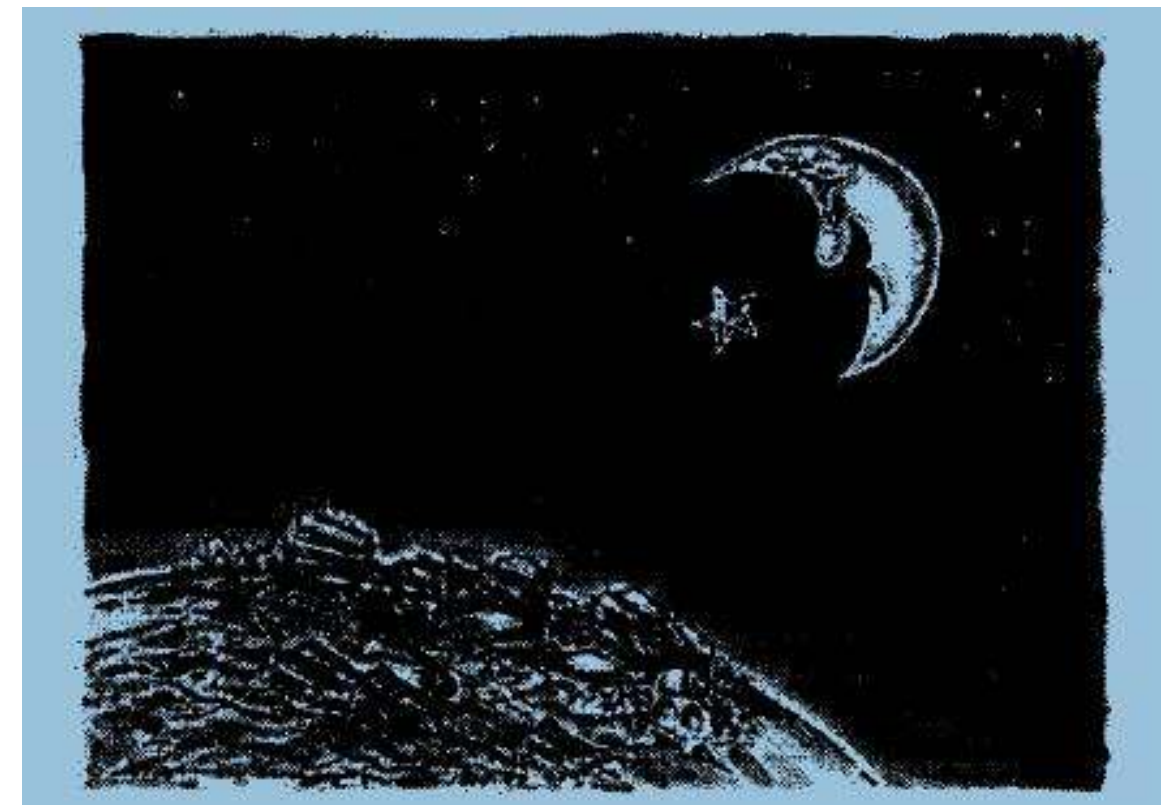

11 Les liens qui se sont manifestés après le tremblement de terre en Turquie se sont renforcés à la suite de celui qui a frappé la banlieue d'Athènes le 7 septembre qui, bien que plus faible en intensité, a donné une fois de plus aux Turcs et aux Grecs le sentiment d'avoir la même destinée, d'où l'idée du partage d'une même géographie en tant que paramètre inchangeable dans les relations gréco-turques ${ }^{20}$. Depuis ces deux séismes, des initiatives bilatérales de coopération, d'aide aux victimes, mais aussi, plus généralement, de paix se multiplient. Peut-on dire que les opinions publiques ont devancé les pouvoirs politiques dans la quête de normalisation de relations de (bon) voisinage? Ce serait assez facile de tirer la conclusion habituelle qui voudrait que les peuples aspirent à la paix et que seuls les gouvernements maintiennent artificiellement la tension. Non seulement sans les points de rupture politiques que j'ai mentionnés plus haut, le point de rupture populaire aurait du mal à avoir lieu, mais de plus, des évolutions positives plus lentes, qui ont traversé la dernière décennie, prouvent que la détente s'est accompagnée d'une véritable volonté politique. Il est vrai qu'en Grèce la société civile s'était mobilisée spontanément en faveur des Turcs, mais depuis l'affaire Öcalan déjà, les initiatives politiques, en partie dues au nouveau ministre des Affaires étrangères Giorgos Papandréou, avaient permis des améliorations sensibles.

\section{Évolutions}

\section{Les Turcs de Grèce vont mieux}

12 Il est assez curieux de constater qu'à chaque période de détente, les améliorations commencent par les problèmes des minoritaires, ce qui prouve que leur résolution ne mobilise pas autant d'énergie qu'il n'y paraît, ou du moins est considérée plus « facile » par les décideurs. Les premiers pas dans ce domaine sont venus de Grèce. Les élections de juin 1989 avaient mis fin à une décennie de pouvoir du PASOK entaché par les scandales financiers comme celui de Koskotas. Après une instabilité politique d'un an et trois élections successives, c'est seulement en avril 1990 que la Nea Demokratia a pu 
former un gouvernement sous la direction de Constantin Mitsotakis avec pour slogan la katharsis (nettoyage, purification). En politique étrangère, plusieurs défis attendaient le gouvernement compte tenu des bouleversements de la conjoncture politique. Le "retour dans les Balkans» de la Turquie obligeait Athènes en quelque sorte à reprendre le dialogue gréco-turc. Au départ, lors de la rencontre des Premiers ministres grec et turc à Londres au sommet de l'OTAN le 6 juillet $1990^{21}$, les deux leaders s'étaient mis d'accord pour la poursuite du dialogue, mais en évitant les sujets qui fâchent comme Chypre et la mer Égée, mais aussi comme les problèmes minoritaires en Thrace occidentale où un mouvement de revendication naissait. Il faut préciser qu'au début des années 1990, c'est la question macédonienne qui avait remplacé la Turquie au rang du principal problème extérieur grec. Malgré la volonté de dialogue de la part d'Athènes, les relations sont entrées dans une phase de tension classique avec la fin de la période d'Özal en Turquie en 1991. C'est donc unilatéralement que la Grèce a commencé à améliorer la situation des musulmans de Thrace occidentale. La décennie 1990 a été témoin d'un réveil nationaliste minoritaire sans précédent dans cette région des Balkans. Les musulmans de Grèce (qui réclament par ailleurs d'être appelés "Turcs») sont devenus de plus en plus revendicatifs et ont été incarnés pour la première fois par un chef charismatique en la personne de Sadık Ahmet. En dépit de problèmes assez graves qui demeurent, un certain nombre de domaines de leur vie quotidienne ont connu des progrès sensibles. Il est vrai que pour ces améliorations, il a fallu attendre la deuxième moitié de la décennie et l'arrivée au pouvoir d'une nouvelle équipe plus « européenne » en Grèce. Il serait injuste de ne pas reconnaître le mérite de ce gouvernement, surtout celui de Giorgos Papandréou en ce qui concerne la minorité de Thrace occidentale.

13 La première amélioration fut le fruit direct de la fin du communisme avec la suppression de la «zone interdite » qui couvrait l'ensemble de la région montagneuse des Rhodopes à la frontière gréco-bulgare. Cette région du nord et nord-ouest de la Thrace occidentale appelée communément « la branche balkanique » abrite la plupart des Pomaks musulmans, une des composantes de la minorité.

L'instauration de cette zone restrictive date d'une décision de 1953 qui s'appuie sur une loi de 1936. À l'origine, son but était d'empêcher les infiltrations probables du communisme en provenance de Bulgarie. C'est une zone qui commence à partir du huitième kilomètre de la frontière gréco-turque, qui s'étend sur 50 kilomètres vers l'ouest avec une largeur de 30 kilomètres approximativement, qui s'arrête au nord de Komotini et de Xanthi. On y trouve 120 villages et une commune importante appelée Echinos. Au total, la population majoritairement musulmane/pomak de la région est estimée à $40000^{22}$. La création d'une telle zone n'a pas été nécessaire à la frontière gréco-turque à cause de l'absence de musulmans dans cette région ${ }^{23}$. Pour les habitants de la zone, l'accès et la sortie se faisaient avec un certificat spécial. Pour les nonrésidents une autorisation du commissariat était obligatoire. De 24 heures à 8 heures, l'accès et la sortie étaient complètement interdits; les observateurs étrangers ont eu du mal à accéder à la zone tout au long des cinquante dernières années.

Les membres de la minorité se plaignaient à juste titre de cette législation archaïque. Selon les plaintes, cette restriction ne visait plus l'infiltration du communisme bulgare, mais la division de la minorité, en empêchant tout contact entre les Turcs des deux grandes villes et les Pomaks des villages des montagnes. D'autant plus que la chute du communisme en Bulgarie à la fin des années 1990 ne rendait plus plausible cette 
argumentation. D'un autre côté, une telle mesure dans un pays faisant partie de l'Europe était mal vue par ses partenaires européens. Finalement, suite à une protestation des habitants d'Echinos, la zone interdite a été abolie en 1995.

La mesure la plus importante touchant de près la vie quotidienne des musulmans de Grèce fut la suppression de l'article 19 du code grec de la nationalité. En effet, jusqu'en 1998 l'article 19 de ce code promulgué en 1955 constituait un des problèmes les plus graves de la minorité. Cet article était le suivant ${ }^{24}$ :

«Si une personne d'origine non grecque quitte le pays sans intention de revenir, elle peut être déclarée comme ayant perdu sa citoyenneté. Cette décision peut également être appliquée aux personnes d'origine non grecque qui sont nées et domiciliées à l'étranger. Les enfants mineurs peuvent être déclarés comme ayant perdu leur citoyenneté si les deux parents ou celui qui est en vie a déjà perdu la sienne. Le Ministère de l'Intérieur décide sur ce sujet en accord avec le conseil national».

Il s'agissait là d'un article discriminatoire, fondé sur des critères raciaux et ethniques qui était, de surcroît, une violation de la Constitution grecque de 1975 elle-même ${ }^{25}$. Il était d'autant plus dangereux qu'aucune précision n'était donnée sur les critères selon lesquels le Ministère décidait que la personne avait quitté le pays sans intention de revenir. Sur le plan international, l'article 19 a été dénoncé pour la première fois en 1990, date à laquelle les pertes de citoyenneté devenaient monnaie courante pour les Turcs qui voyageaient en Turquie, par le rapport des droits de l'homme du Département d'État des États-Unis. D'autres instances internationales ont suivi cet exemple, comme le Parlement européen en $1993^{26}$. Compte tenu de ces pressions, en janvier 1998, les autorités grecques, à l'initiative de Giorgos Papandréou, ont supprimé cet article sans toutefois proposer d'effet rétroactif. Néanmoins, dans les faits, depuis le rapprochement gréco-turc de 1999, les tribunaux grecs acceptent les requêtes individuelles des heimatlos pour retrouver leur citoyenneté et une grande partie de ces requêtes semble aboutir ${ }^{27}$. Si la suppression de l'article 19 est antérieure à la détente gréco-turque, l'attitude souple des tribunaux en est un fruit.

Toujours à partir de la deuxième moitié des années 1990, d'autres évolutions positives touchant la vie quotidienne de la minorité ont eu lieu. En particulier, ce sont les «tracasseries » administratives dont les Turcs de Grèce faisaient fréquemment l'objet dans les années 1980 et au début des années 1990 qui ont peu à peu disparu. Acquisition de biens, obtention de différentes autorisations et licences, obtention des permis de conduire, et autres démarches administratives qui jalonnent la vie d'un individu étaient véritablement jonchées d'embûches ${ }^{28}$. Dans ces améliorations, il faut voir non seulement les fruits d'un rapprochement gréco-turc, car les minorités réciproques ont toujours été tributaires des relations bilatérales ${ }^{29}$, mais aussi les résultats d'une européanisation de l'État grec plus respectueux des particularités et moins réticent à accorder une égalité entre ses citoyens.

\section{Diminution des tensions autour du Patriarcat}

On peut observer une évolution parallèle en Turquie également, même si elle est de moindre ampleur. Les Grecs de Turquie aussi, du moins les institutions qui leur sont en principe liées (cette liaison est de moins en moins évidente à cause de la quasidisparition numérique de la minorité) ont vu évoluer partiellement une partie de leurs 
problèmes: des avancées significatives ont concerné le Patriarcat et les fondations pieuses.

Alors que les Turcs de Grèce étaient en pleine crise des muftis, en octobre 1991, le Patriarche Dimitrios $1^{\text {er }}$ est décédé, remplacé par Vartholoméos $1^{\text {er }}$. Son élection par le Saint Synode s'est déroulée pour la première fois sans que les autorités turques interviennent par le biais de la préfecture d'Istanbul, comme cela était l'usage depuis la fondation de la République turque. Le Président de la République d'alors, Turgut Özal, ne voulait pas avoir d'ennuis à cause de cette élection. Depuis 1991, le nouveau Patriarche Vartholoméos, a réussi à ressusciter le Patriarcat, avec l'aval implicite des autorités turques, et à internationaliser l'institution qui était tombée en désuétude. Au niveau international la tâche du nouveau Patriarche était ardue. Avec la chute du bloc soviétique, la donne était changée dans l'équilibre politique Est-Ouest, mais aussi dans le monde orthodoxe. En Russie, un réveil du nationalisme russe s'accompagnait d'un réveil spirituel et l'orthodoxie était redevenue un des ingrédients indispensables de l'identité russe. Par ailleurs, les peuples orthodoxes de l'ancien bloc soviétique tentaient de s'affranchir par tous les moyens de la suprématie russe, y compris dans les domaines religieux, et certains n'ont pas hésité à chercher le soutien du Phanar. Et enfin, le nouveau Patriarche a accru les relations avec le mouvement œcuménique, mais aussi avec le Vatican, en reprenant ces relations-là où les avait laissées Athénagoras.

21 Cette internationalisation a naturellement mis à l'ordre du jour la question de l'œcuménicité du Patriarcat. Il faut préciser que Vartholoméos $1^{\mathrm{er}}$, dans ses activités internationales a trouvé plusieurs alliés en Turquie qui voyaient en un Patriarcat fort un moyen d'accroître le prestige du pays, et un moyen d'influer sur les champs d'action patriarcaux. À l'extérieur également, le Patriarcat jouit dorénavant d'un prestige et d'un soutien notamment aux États-Unis et dans l'Union européenne. Certes, d'autres milieux turcs développent comme à l'accoutumée les thèses d'un complot accusant le Phanar de poursuivre un but sécessionniste, mais d'une manière générale, on peut dire que la situation du Patriarcat est bien plus favorable à un développement futur qu'il y a une décennie ${ }^{30}$.

Une autre question qui a sensiblement évolué est celle des fondations pieuses. Depuis 1974, les vakıfs, un des piliers des institutions minoritaires étaient menacés. Immédiatement après l'intervention turque à Chypre, la Direction générale des Fondations pieuses (Vakıflar Genel Müdürlügü), en accord avec les services du premier ministre, prit la décision de demander que tous les biens acquis par les fondations pieuses non-musulmanes par quelque moyen que ce soit (héritage, donation, achat, etc.), et ce à partir de 1936, soient rendus à des ayants droit. À cette date selon la loi $n^{\circ} 2762$, l'État turc avait demandé à toutes les fondations pieuses non-musulmanes de faire une déclaration exhaustive de leurs biens immobiliers ${ }^{31}$. Cette liste désormais connue sous le nom de " 36 Beyannamesi » (la Déclaration de 1936) a été établie et communiquée aux autorités et... oubliée pendant près de 40 ans. C'est seulement en 1974, donc immédiatement après l'intervention turque à Chypre, qu'elle revient à l'ordre du jour. Sous prétexte que les fondations en question n'ont pas précisé dans leur déclaration de 1936 qu'elles pouvaient dans le futur accepter des donations ou acquérir d'autres biens immobiliers, tous les biens acquis de 1936 à 1974 ont été considérés comme caducs. Sur le moment cette décision n'a pas eu beaucoup d'écho, on peut dire que dans l'euphorie générale de l'intervention turque à Chypre, cette mesure est passée 
totalement inaperçue. Même les journaux minoritaires n'en ont pas parlé32. C'est seulement par la suite qu'on y revint, lorsque les fondations ont commencé à perdre progressivement leurs biens immobiliers au profit des héritiers présumés ou réels de la personne qui détenait le bien avant la fondation, ou au profit du Trésor public (Hazine), ou au profit de la Direction des biens immobiliers nationaux (Milli Emlak) en cas d'impossibilité de trouver les héritiers. Ces deux dernières organisations sont d'ailleurs en concurrence. Les responsables des fondations pieuses se sont rendu compte de la gravité de la situation une ou deux années plus tard, lorsque les procès ont commencé à pleuvoir. Le problème des biens des fondations minoritaires a été le principal souci des Grecs, des Arméniens et des Juifs de Turquie jusqu'en 2003. À cette date, le 8 août 2002 plus exactement, parmi une série de réformes dans le cadre de l'intégration européenne, trois décisions déterminantes ont été prises. Il s'agit de l'abolition de la peine de mort, de l'autorisation d'enseignement des langues minoritaires et de l'autorisation pour les fondations minoritaires d'acquérir de nouveaux biens sous certaines conditions. Cette nouvelle loi reconnaissait implicitement qu'auparavant ces acquisitions étaient impossibles. Les Grecs de Turquie, avec les autres minoritaires, ont été très contents de cette avancée significative, bien que le décret d'application paru en octobre soit l'objet de controverse ${ }^{33}$.

\section{La société civile : initiatives et attitudes}

23 Il suffit de regarder globalement l'ensemble des évolutions positives, pour y déceler l'influence de l'achèvement de l'européanisation de la Grèce comme la perspective d'une intégration européenne de la Turquie ${ }^{34}$. C'est dans ce cadre qu'il faut considérer la construction d'une mosquée à Athènes ${ }^{35}$, projet soutenu par ailleurs par le Patriarche Vartholoméos $^{36}$, mais aussi l'autorisation d'une chaine de télévision musulmane (turque) en Grèce. Ces évolutions ne sont pas uniquement dues aux décisions et politiques gouvernementales. L'émergence dans les deux pays d'une société civile plus ou moins indépendante du pouvoir joue un rôle non négligeable également. Un réseau d'associations, d'organisations non gouvernementales, d'institutions socioprofessionnelles, et de divers groupements d'intérêt semble parfois en avance dans la quête d'une normalisation des relations entre les deux États, et plus encore, entre les deux peuples. Ces regroupements servent de deux manières. D'une part, ils permettent la transformation lente, mais solide des opinions publiques avec des activités s'adressant directement à elles, et d'autre part, ils s'associent dans les activités culturelles, économiques et académiques en préparant le terrain à des initiatives plus politiques.

Par exemple, les initiatives émanant d'universitaires ou d'étudiants ${ }^{37}$ des deux pays donnent des résultats surprenants. À ce propos, il faut signaler deux actions officielles concernant les livres scolaires. Alors qu'en Turquie une réforme des livres d'histoire est en cours pour y supprimer le "discours de haine" envers les Grecs ${ }^{38}$, en Grèce, l'Université d'Athènes travaille actuellement sur un projet européen de préparation de manuels scolaires spéciaux pour les écoles primaires minoritaires en Thrace. À cette liste, il faut ajouter des initiatives privées d'hommes et de femmes, des deux côtés de la mer Égée, qui animent des forums notamment à travers des sites Internet. J'ai pu compter une trentaine de sites de ce genre où des projets de rapprochement sont élaborés. Depuis les deux tremblements de terre, le nombre de ces sites augmente à une vitesse vertigineuse. 


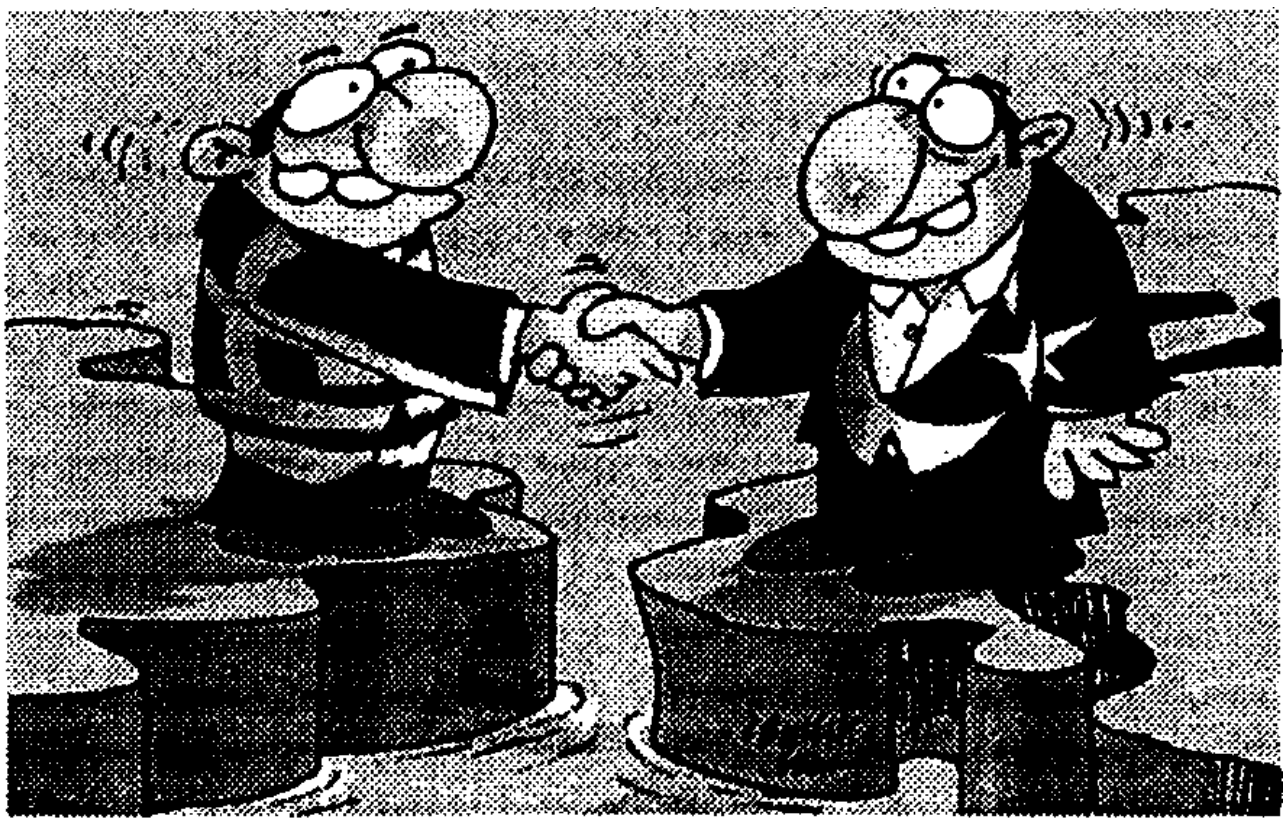

http://members.aol.com/aegfriends/apeace.html

Les concerts communs de musiciens turcs et grecs sur les traces de Mikis Théodorakis et Zülfü Livaneli à l'instar de Burak Kut et Sakis Rouvas ou Sezen Aksu et Haris Alexiou, ou tout simplement des activités communes de loisirs telle cette rencontre de Ferrari grecques et turques ${ }^{39}$ (!), donnent des couleurs à des coopérations plus sérieuses entre hommes d'affaires ${ }^{40}$, financiers ${ }^{41}$, voyagistes $^{42}$, journalistes ou diplomates. Il ne faut pas oublier que, de tout temps, même aux moments des crises intenses, des efforts d'amitié et de rapprochements ont été déployés par ce genre de groupes, mais ces initiatives se multiplient d'une manière considérable et trouvent de plus en plus d'échos dans les médias qui, jusque-là avaient une approche belliqueuse. Ainsi, la normalisation des relations devient réalisable, souhaitable et, le plus important, anodine ${ }^{43}$.

\section{Permanences}

\section{Chypre}

Les deux premiers chapitres de cet article brossent incontestablement un tableau optimiste. Il n'est pas difficile de vérifier que cette situation positive est réelle, bien que non définitive. Car il est temps de la nuancer par la description de quelques domaines dans lesquels les continuités résistent aux points de rupture sans toutefois être imperméables à toute évolution. Un certain nombre de problèmes bilatéraux ne sont toujours pas résolus et continuent à empoisonner les relations en restant des causes de tensions futures. Bien entendu, en tête de ces "problèmes" non résolus vient la question chypriote, qui est, selon la majorité des observateurs, non seulement le catalyseur des autres différends, mais de plus, demeure la cause principale de l'ensemble du conflit gréco-turc. Bien que je ne partage pas cet avis ${ }^{44}$, il est indéniable que la résolution pacifique de la question chypriote influencerait plus que 
favorablement les relations gréco-turques dans d'autres domaines, ne serait-ce que par son impact sur les opinions publiques.

Il n'est pas question ici de détailler toutes les péripéties de cette question complexe durant la dernière décennie du $\mathrm{xx}^{\mathrm{e}}$ siècle. Je ne ferai que quelques constats. L'ensemble des points de rupture analysés ci-dessus concerne évidemment la question chypriote également. Mais sur cette question d'autres facteurs entrent en jeu. Le premier de ces facteurs est l'européanisation du conflit. En ce sens, il faut constater la réussite de la politique grecque consistant à transposer les problèmes bilatéraux sur un terrain qui lui est plus favorable, celui de l'Europe ${ }^{45}$. C'est ainsi que dès les débuts des années 1990, Chypre est devenue une des variables importantes des relations turco-européennes. C'est en juillet 1990 que la République de Chypre s'est portée candidate pour entrer dans l'Union européenne avec le soutien de la Grèce et à la grande stupéfaction de la Turquie. Cette demande a non seulement suscité des commentaires enflammés des autorités turques, mais de plus, a contribué au resserrement des relations entre la Turquie et la "République Turque de Chypre du Nord». Selon les autorités turques, mais aussi selon Rauf Denktaş, la candidature de la République de Chypre à l'Union européenne était politiquement et juridiquement impossible. Politiquement impossible, parce que l'administration grecque de lîle ne représente pas les Turcs de l'île, communauté constitutionnelle depuis 1960, alors que la demande d'adhésion a été faite au nom de la République de Chypre, parce qu'une adhésion sans une solution fédérale est inacceptable, dans la mesure où, dans l'état divisé de l'île, les règles communautaires sont inapplicables, mais aussi parce qu'en cas d'adhésion, de facto, les Grecs auraient deux voix dans la communauté. Cette demande était également juridiquement illégale, selon les responsables turcs. Parce que la Constitution chypriote de 1960 interdisait l'unification de l'île avec un autre État (article 185), mais aussi selon une interprétation de l'article 50 de la même constitution, parce que Chypre ne peut adhérer à une organisation quelconque dont la Grèce et la Turquie ne seraient pas membres $^{46}$.

Malgré les protestations turques (d'Ankara et de Nicosie ${ }^{47}$ ), la candidature chypriote fut bien accueillie par l'Union européenne. Cette candidature a eu, bien entendu, des conséquences multiples. Outre le resserrement des relations entre la Turquie et le nord de l'île déjà mentionné, elle fut un des catalyseurs des efforts onusiens pour le règlement du conflit. Ces efforts commencés par «la suite d'idées" de Boutros Ghali ont pris une ampleur sans précédent au tournant du siècle avec les initiatives de Kofi Annan qui ont débouché sur un nouveau plan. Mais, entre-temps, la sclérose de la question chypriote est apparue à travers une crise multiforme, celle des S-300. En janvier 1997 ces missiles russes achetés par les Chypriotes grecs n'ont pas pu être installés dans l'île en raison des pressions et menaces de la Turquie. Cette crise démontrait que Chypre restait un déclencheur potentiel de conflit armé et que son règlement était indispensable.

conflit. En juillet 1998, la Cour européenne des Droits de l'Homme de Strasbourg a condamné la Turquie à verser des indemnités à Titina Loizidou, une Chypriote grecque qui accusait le gouvernement turc de l'empêcher de se rendre chez elle. Cette décision contraignante a mis la Turquie dans l'embarras, car verser la sanction revient à reconnaître que le nord de Chypre est occupé par l'armée turque et il existe des centaines de dossiers du même genre qui attendent devant la Cour. Le paiement a finalement été fait en décembre 2003 avec, semble-t-il, des garanties de la Cour quant 
aux autres dossiers en attente. Au-delà de la signification juridique, ce fait démontrait combien les politiques turques concernant Chypre se trouvaient dans une impasse et combien l'absence de solution, donc le statu quo, ne constituait plus une solution.

Il est étonnant de constater que, malgré ces développements inquiétants et malgré l'acceptation des négociations intercommunautaires du bout des lèvres à partir de 1997, la situation n'a que peu évolué. Il faut dire que le sud de Chypre avait trouvé un appui considérable dans la perspective d'une adhésion européenne. Mais il est vrai aussi que l'UE préférait de loin accueillir une Chypre (ré)unie, de quelque façon que cela soit. Mais l'irruption de l'Europe comme facteur déterminant dans la résolution de l'affaire chypriote a ajouté un nouveau paramètre à la situation. Alors que jusque dans les années 1990, celle-ci dépendait des relations gréco-turques (et vice versa), à partir de la deuxième moitié de la décennie, elle dépend aussi des relations turcoeuropéennes (et vice versa?). Ainsi, même si les discours émanant des deux côtés (instances européennes et autorités turques) font une distinction claire entre le processus de réunification chypriote et celui de l'intégration turque, dans les faits, il est évident que ces deux processus sont intimement liés ${ }^{48}$.

31 La perspective européenne conjuguée à une crise économique et sociale ainsi qu'une structure étatique maladive ont fait qu'au nord de Chypre, d'autres interlocuteurs sont apparus; le renforcement de la société civile s'est accompagné d'un durcissement de l'opposition à la politique de Denktaş, créant un climat de mécontentement à la RTCN. Ce climat a été compris par le nouveau pouvoir en Turquie, celui de l'AKP (Adalet ve Kalkınma Partisi), des islamistes dissidents du Parti de la Prospérité. En effet, les premiers discours annonçaient un changement radical dans l'attitude de la Turquie visà-vis du problème chypriote. C'est vrai que ce discours a été dilué sous la pression de l'armée, des milieux nationalistes ainsi que de Denktaş et c'est en ce sens que nous pouvons parler d'une continuité. Néanmoins, l'intensification de la pression populaire au nord de Chypre ${ }^{49}$ consécutive à la concrétisation de l'adhésion chypriote à l'Union européenne en 2003 a eu des conséquences inattendues, avec des manœuvres tactiques des administrations turque et grecque de l'île dans le cadre de la décision de la « libre » circulation pour les deux peuples. 


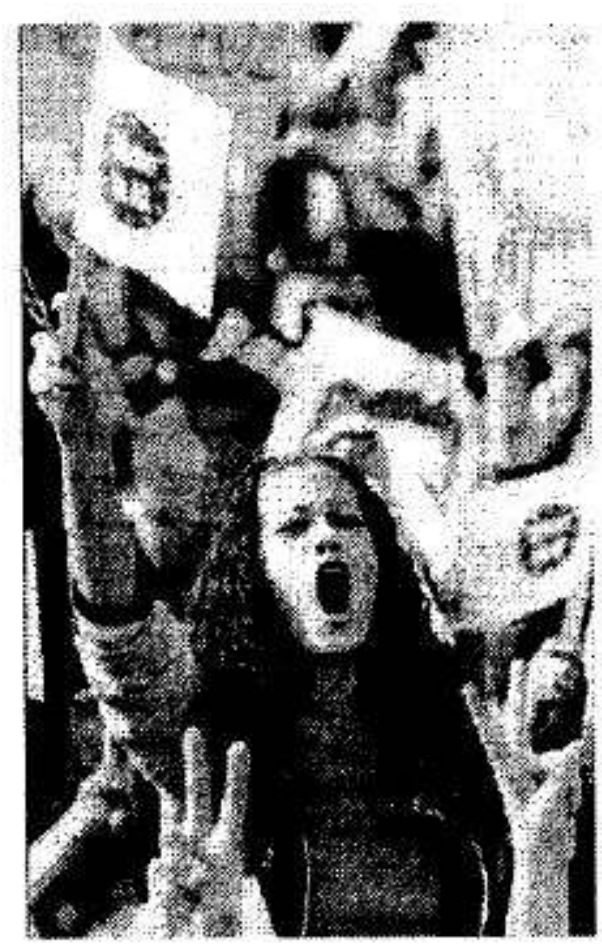

32 Cela dit, force est de constater que malgré ces évolutions, malgré les efforts onusiens et même malgré un changement sensible des attitudes des mères patries, Chypre reste divisée ${ }^{51}$. La volonté d'Ankara d'utiliser la question chypriote dans sa propre adhésion à l'Union fait craindre des revirements, même si la volonté des Chypriotes turcs de faire également partie de l'Europe pèse sur les attitudes ${ }^{52}$.

\section{Problèmes égéens}

Indépendamment des problèmes chypriotes, d'autres conflits liés à la mer Égée, continuent à exister quasiment sans développement notable. Les deux seuls changements que la dernière décennie a vus sont un ajout à la liste des points conflictuels, celui des «zones grises » et un nouvel acteur potentiel dans la résolution éventuelle du conflit, l'Europe. À l'instar du problème chypriote, la candidature turque à l'Union européenne a forcé celle-ci à s'intéresser au conflit égéen. Dans la déclaration d'Helsinki, en décembre 1999, il a été décidé que si les deux protagonistes n'arrivaient pas à se mettre d'accord avant 2004 sur les problèmes égéens, ce serait la Cour Internationale de La Haye qui trancherait. Il est évident que cette solution, jamais acceptée par la Turquie, mettait Ankara devant un fait accompli. Ainsi, la résolution du contentieux égéen était placée parmi les objectifs à moyen terme. En fait, cette solution ne plaisait pas beaucoup à la Grèce non plus qui ne reconnaît pas d'autres problèmes que celui du plateau continental ${ }^{53}$.

34 En attendant, les frictions habituelles ont continué, notamment les plaintes mutuelles de violation de l'espace aérien. À chaque friction, le climat de rapprochement actuel se trouve en danger, les discours et attitudes étant stéréotypés ${ }^{54}$. En mai 2003, la presse turque rapportait toujours les propos de Simitis et de Giorgos Papandréou concernant la permanence de la politique égéenne grecque $^{55}$, auxquels répondaient les 
responsables turcs par des propos identiques. L'aspect technique du conflit empêche des avancées, car les politiques et les discours qui les soutiennent sont depuis trop longtemps figés, à tel point que les décideurs se sentent prisonniers à la fois de leurs propres discours antérieurs et des réactions des opinions publiques éventuelles à venir. Ce point précis du conflit bilatéral n'a pu jusque maintenant profiter ni des ouvertures réelles faites de concessions mutuelles, ni de la « diplomatie du Kokoreç $~_{56}$ teintée de populisme.

Les différends égéens ne sont pas imaginaires. Il s'agit là d'un conflit de frontières classique, presque archaïque dans ses tenants et aboutissants. C'est le conflit le plus technique, complètement déshumanisé dans la mesure où il ne concerne directement aucun groupe humain, sauf peut-être quelques pêcheurs qui ont tout à gagner en cas d'une solution équitable ${ }^{57}$. C'est dans ce sens que ce conflit de souveraineté reste le moins passionnant, sauf pour juristes et géopolitologues, mais il est révélateur d'un certain état d'esprit des autorités des deux pays.

\section{Minorités}

En ce qui concerne la question des minorités « réciproques ", en dépit des évolutions mentionnées ci-dessus, il existe deux domaines où les situations restent bloquées: domaine religieux pour les musulmans de Grèce et domaine éducatif pour les Grecs de Turquie. Il est évident que la question des muftis de Thrace occidentale dépasse largement la simple liberté de culte. Rappel des faits :

Selon le traité de Lausanne de 1923, les musulmans de Thrace occidentale possèdent des droits familiaux musulmans concernant le mariage, l'héritage, le divorce, etc. Dans ce cadre, les deux muftis, de Komotini et de Xanthi, ont, à côté de leur rôle religieux, des prérogatives juridictionnelles. Ces deux muftis ont toujours été nommés par les autorités grecques et jusque dans les années 1980 cela convenait très bien à la minorité. C'est dans ces années d'éveil nationaliste minoritaire, notamment derrière une figure emblématique qu'est Sadık Ahmet ${ }^{58}$ que sont morts, l'un après l'autre, les deux muftis en question. Le 2 juin 1985, le mufti de Komotini, Hüseyin Mustafa mourut à 73 ans d'une crise cardiaque. ${ }^{59}$ Une panique totale s'empara de l'élite de la minorité. En effet, rien n'était prévu pour le remplacement du mufti. Il est vrai qu'à cause des relations familiales et clientélaires habituelles qui régissent les institutions minoritaires, le gendre du mufti défunt se voyait déjà à ce poste avant le décès de son beau-père. Les événements se compliquent avec la nomination d'un prêcheur comme mufti provisoire par le préfet de Komotini. Le gendre, Hasan Paçaman, et ceux qui le soutenaient se sont immédiatement opposés à cette nomination, prétextant que cet homme avait fait des études en Égypte et l'accusant d'être proche des pays arabes et du pouvoir grec $^{60}$. Un vide de sept mois a suivi la démission, sous les pressions, du mufti par intérim désigné. Pendant ces mois une partie des notables minoritaires s'est mise d'accord pour l'élection d'un mufti selon une loi datant de 1920, considérée comme caduque par les autorités grecques. C'est à ce moment-là qu'un autre imam, Cemali Meço, considéré comme proche des pays arabes, fut nommé mufti par intérim ${ }^{61}$. Alors qu'une partie de l'intelligentsia minoritaire soutenait activement ce dernier, les plus proches de la Turquie s'y sont opposés vigoureusement. Une campagne de presse déchaînée a été lancée contre le nouveau nommé. La nomination de Cemali Meço prend une autre tournure dans les années 1990. Le 3 novembre 1988, le gouvernement grec présente un 
projet de loi à l'assemblée. Selon ce projet, les muftis par intérim devenaient automatiquement muftis en titre et les deux muftis des musulmans devenaient des fonctionnaires attachés aux préfets. Alors que les tensions étaient encore très vives, le mufti de Xanthi meurt en février 1990. Immédiatement après ses funérailles à Istanbul le 15 février, auxquelles 10000 personnes ont assisté, pour ne pas subir le même fait accompli qu'à Komotini cinq ans auparavant, les leaders de la minorité se réunissent et préviennent le gouvernement que la minorité ne reconnaîtra pas un autre mufti nommé. Ainsi, le 17 août 1990, dans 52 mosquées de Xanthi, il y eut des élections clandestines et Mehmet Emin Aga, fils du mufti défunt, fut élu mufti de Xanthi. Le 15 décembre 1990, le comité de prêche prend cette fois la décision d'organiser les élections d'un mufti à Komotini. À l'issue de l'élection du 28 décembre, toujours à main levée, Ibrahim şerif est déclaré mufti élu de Komotini contre le mufti nommé Cemali Meço. Le gouvernement grec réagit seulement le 22 août 1991, en nommant Mehmet Emin Sinikoglu comme mufti de Xanthi. Actuellement la même situation se poursuit. À Komotini et à Xanthi existent toujours deux muftis. Les défenseurs des muftis élus ne reconnaissent pas les muftis nommés et vice versa et ce malgré les condamnations du gouvernement grec par la Cour européenne des Droits de l'homme ${ }^{62}$.

Il est évident que les musulmans de Thrace ont d'autres problèmes qui demeurent intacts comme ceux concernant les écoles, les fondations pieuses, etc. Néanmoins, c'est la question des muftis qui est la plus emblématique des permanences en ce qui les concerne. En revanche, pour les Grecs de Turquie, les questions éducatives sont les plus brûlantes, objets de plus de plaintes. D'une manière générale, les écoles minoritaires grecques en Turquie souffrent du manque d'élèves et d'une suspicion permanente des autorités turques à leur égard ${ }^{63}$. Je voudrais mentionner ici un problème annexe, mais symptomatique. C'est le cas particulier de l'École théologique de Halki ${ }^{64}$.Dans le cadre de son rôle de formation du personnel religieux, c'est une des institutions les plus importantes, indispensable pour la survie du Patriarcat. Cette école qui a été fondée en 1844, avait pour mission, outre cette formation, de dispenser un enseignement théologique orthodoxe unificateur face aux courants nationalistes dans le monde orthodoxe. Après de multiples changements de statuts et de niveaux, en 1951, un enseignement de lycée de 4 ans y est délivré, suivi de 3 ans de théologie. L'École est sous l'administration directe du Patriarcat et le directeur de l'école est choisi parmi les Métropolites. Tout au long de son fonctionnement, l'école a accueilli des étudiants de Turquie, mais aussi des Églises étrangères comme celle d'Angleterre ou encore d'Éthiopie.

Dans le cadre de l'interdiction des universités et académies privées en 1971, l'École théologique de Halki a été fermée suite à la décision de la Cour constitutionnelle relative «à l'annulation de certaines clauses de la loi $\mathrm{n}^{\circ} 625$ relative aux établissements d'enseignement privé $»^{65} . \mathrm{Il}$ faut signaler ici une erreur commise par la plupart des observateurs, qu'ils soient Grecs ou Turcs, concernant cette fermeture. Celle-ci n'a pas été une décision sans appel prise par le gouvernement turc comme on peut souvent le lire. Il s'agit plutôt d'une décision de cessation d'activité (et non de la fermeture administrative) prise par les instances patriarcales, pour ne pas continuer les activités de cette école sous le contrôle du ministère de l'Éducation nationale turque. Durant les 127 années où l'école était en activité, 930 théologiens et cadres religieux y ont étudié. Trois cent quarante-trois de ces diplômés sont devenus des évêques dont 12 ont été élus Patriarche ${ }^{66}$. Ces chiffres confirment l'importance de cette institution tant au niveau administratif que religieux et psychologique. Certes, il existe actuellement une école 
théologique à Boston, mais elle est très loin de satisfaire les besoins du Patriarcat. Les gouvernements turcs, pour leur part, donnent à la fois des signes d'espoir pour une réouverture éventuelle et demandent en même temps des contreparties en Thrace occidentale ${ }^{67}$, comme s'il ne s'agissait pas à Heybeliada de citoyens turcs. Toute activité en faveur ou en défaveur de la minorité est jugée selon les critères d'intérêts 'nationaux', ainsi la réouverture de cette école.

Ces derniers temps, l'École théologique de Halki est revenue à l'ordre du jour, notamment avec les pressions de plus en plus nettes de l'administration américaine pour sa réouverture. En effet, parallèlement au regain d'importance du Patriarcat depuis le début des années 1990 aux yeux de Washington, de très hautes personnalités américaines se sont exprimées en faveur d'un "geste " d'Ankara permettant la réouverture de cette école. Que cela soit l'ancien secrétaire d'État américain Madeleine Albright ${ }^{68}$ ou même l'ex-président américain Bill Clinton ${ }^{69}$, ils ont à plusieurs reprises exprimé publiquement ce souhait. Ce qui est étonnant, compte tenu de la rivalité entre l'Église de Grèce et le Patriarcat, c'est de voir à quel point la Grèce soutient cette réouverture. Déjà en février 1992, le premier ministre grec s'adressait directement au premier ministre turc pour demander son autorisation ${ }^{70}$. Mais, surtout après le rapprochement gréco-turc de 1999, cette demande s'est inscrite parmi les gestes qu'Athènes demandait à Ankara en contrepartie de son soutien à la Turquie dans son processus d'intégration européenne. À première vue, cette insistance de la part d'Athènes est étonnante, mais une analyse plus fine suffit pour la comprendre. À partir de 1950, le gouvernement turc avait permis l'inscription d'étudiants étrangers dans cette école. De 1950 à 1969 (date à laquelle le dernier ressortissant étranger a quitté l'école), l'École théologique de Halki a accueilli 225 étudiants dont seulement 38 étaient des Grecs de citoyenneté turque et, parmi les 187 ressortissants étrangers, 162 étaient des citoyens helléniques. Et ce, pendant une période où les Grecs de Turquie étaient nombreux. Ainsi, si aujourd'hui cette réouverture était assurée, compte tenu du nombre et de la pyramide des âges des Grecs citoyens turcs, naturellement beaucoup de Grecs de Grèce viendraient y étudier. C'est, entre autres, cet aspect des choses qui suscite beaucoup de réactions en Turquie. Parmi les milieux qui soutiennent cette réouverture, nous voyons les Fethullahçı, une des branches de la confrérie turque Nurcu qui prônent le dialogue interreligieux, et une partie des intellectuels qui estiment que l'amitié gréco-turque est indispensable d'une part et que les diplômés de cette école vont redorer l'image de la Turquie. On parle même de «l'exportation de dignitaires religieux $»^{71}$. En face, nous voyons des opposants farouches à cette réouverture. On peut y distinguer deux groupes, les nationalistes turcs qui considèrent que cette école deviendra à nouveau un nid d'espions nuisibles à l'État turc, et les kémalistes ultra-laïcs qui considèrent que si cette réouverture se fait, d'autres communautés, musulmanes cette fois-ci, vont réclamer l'ouverture d'écoles théologiques islamiques autonomes, justifiant ainsi clairement le soutien de Fethullah Gülen à cette réouverture. Une proposition de compromis semble avoir été faite par le gouvernement turc. Il s'agit de la réouverture de l'École théologique de Halki, mais attachée à une "Haute Section des Cultures et Religions " attachée elle-même à la Faculté de Théologie musulmane de l'Université d'Istanbul ${ }^{72}$. Le Patriarche lui-même ne serait pas indifférent à cette proposition, bien qu'une dépendance vis-à-vis du YOK (Yüksek Ogrenim Kurumu), Institution de l'Éducation supérieure, qui chapeaute l'ensemble des universités et écoles supérieures turques le gênerait évidemment. Mais 
une partie de son entourage, traditionaliste et nationaliste, s'y oppose formellement et réclame une autonomie totale pour l'École. Donc pour l'instant l'affaire est bloquée.

Le 30 octobre 1998, le gouvernement turc a unilatéralement révoqué les membres laïcs du conseil d'administration de la fondation pieuse de laquelle dépend l'École de Halki ${ }^{73}$. Cette révocation a suscité la colère du Patriarcat et du gouvernement grec. Vartholoméos, d'habitude très réservé à l'égard du gouvernement turc, a considéré cela comme une atteinte aux droits minoritaires et a déclaré aux fidèles pendant une messe à l'église d'Arnavutkoy: "Vos droits sont bafoués " ${ }^{74}$. Quant au gouvernement grec, cette révocation a été utilisée pour démontrer aux partenaires occidentaux qu'Ankara maltraite toujours les Grecs de Turquie ${ }^{75}$. Le gouvernement turc a justifié cet acte lors d'une conférence de presse du ministère des Affaires étrangères (!), en précisant que les membres en question violaient les lois sur les fondations pieuses, "parce qu'ils utilisaient les bâtiments et les terrains de l'école pour des activités autres que scolaires $»^{76}$. Certains ont interprété cette décision comme un prélude à la fermeture définitive et administrative de l'école, d'autres au contraire, ont estimé qu'il s'agissait pour Ankara de placer des gens proches du gouvernement avant la réouverture. Quoi qu'il en soit, lors de ma visite à l'École théologique de Halki, j'ai pu constater l'état impeccable du matériel, des bâtiments, comme si demain elle pouvait accueillir des étudiants. Cela montre que cette réouverture est non seulement attendue, mais également préparée.

\section{Conclusions}

Les ruptures, les évolutions et les permanences évoquées dans cet article sont dans la nature des choses. Même les problèmes qui semblent les plus figés connaissent des mutations, certains problèmes trouvent des issues plus ou moins durables et d'autres, nouveaux, s'ajoutent au conflit. Mais il est indéniable que nous vivons, depuis la fin de la décennie 1990 une période propice aux évolutions positives dans toutes les composantes du différend gréco-turc. Néanmoins, il ne faut pas oublier qu'il ne s'agit pas d'une première. D'autres périodes de détente ont eu lieu, parfois plus explicites que la période actuelle, mais toutes se sont achevées par des tensions graves. La période de relations chaleureuses commencée par le Traité d'Ankara de 1930 s'est altérée pendant la Seconde Guerre mondiale. Celle qui a commencé à la fin de cette guerre grâce à la politique américaine dans la région s'est brusquement terminée en 1955, avec l'apparition du problème chypriote. L'« esprit de Davos » débuté en 1988 s'est peu à peu éteint quelques années plus tard ${ }^{77}$. On aura tort de prendre ces quelques dernières années comme une étape définitive vers un règlement global du différend gréco-turc. Car les raisons de ces conflits, liées aux mémoires collectives des deux nations, restent profondes. Cela dit, la période actuelle a une particularité et ce à trois niveaux. La fin de la guerre froide et la perspective européenne offrent un cadre favorable au développement des relations bilatérales et multilatérales au niveau macropolitique. Au niveau local, la Grèce se débarrasse peu à peu de ses démons nationalistes dont le paroxysme a été atteint avec la junte des colonels, pour devenir un État européen au sens plein du mot. Quant à la Turquie, empêtrée dans des problèmes économiques et sociaux, elle semble néanmoins posséder maintenant un cadre démocratique plus solide et une stabilité politique relative. Et enfin, les mémoires collectives dont il est fait mention semblent ne plus empêcher, du moins provisoirement, les opinions 
publiques de se sentir davantage ouvertes. Ainsi, il y a peut-être une porte entrouverte pour que la période de bonnes relations actuelles soit durable et permette des évolutions positives.

Concernant les relations bilatérales, le tableau de ces dernières années est néanmoins positif. Seulement depuis quatre ans, 22 accords bilatéraux de diverses natures ont été signés. Parmi ces derniers, ceux concernant les coopérations économiques semblent les plus importants. Par exemple, les investissements pour empêcher la double imposition des marchandises atteignent les 600 millions de dollars ${ }^{78}$. Le volume de commerce atteint désormais les deux milliards de dollars par an. La construction d'un pipeline de $280 \mathrm{~km}$ pour le transport du gaz naturel de la Caspienne vers l'Europe à travers la Turquie et la Grèce a débuté.

$\mathrm{Au}$ moment où ces lignes sont rédigées, des développements importants ont eu lieu en Grèce et à Chypre. En Grèce, dans la période préélectorale, pour la première fois, les relations avec la Turquie n'ont pas été au coeur de la campagne électorale. La défaite de Giorgos Papandréou, un des artisans de la normalisation spectaculaire des relations de ces quelques dernières années, n'a pas empêché la poursuite des efforts de rapprochement. D'ailleurs, ce n'est pas un hasard que la campagne interne du PASOK a commencé par une visite de Papandréou en Thrace occidentale ${ }^{79}$. Inutile de dire qu'il y a été accueilli chaleureusement par les Turcs, d'autant plus que Papandréou y a proposé une réduction mutuelle équilibrée des forces armées. Kostas Caramanlis, actuel Premier ministre avait également donné des gages à la minorité turque ${ }^{80}$. Dans ce cadre, la Grèce continuera certainement à jouer un rôle prépondérant dans les relations turco-européennes. Concernant les minorités réciproques, un assouplissement est visible des deux côtés. Ainsi, le Patriarche Vartholoméos a pu se rendre sans problème à Cuba pour y inaugurer la première église orthodoxe depuis la révolution, sans que cela pose un problème aux autorités turques qui, dans le temps, s'opposaient farouchement à ce genre d'initiatives, considérant le Patriarcat comme une Église interne à la Turquie ${ }^{81}$.

Certes, les problèmes égéens ne sont pas encore résolus. Les deux parties tiennent secret le déroulement des pourparlers qui durent depuis deux ans sur cette question. Néanmoins, les avancées sont indéniables. N'oublions pas qu'en 1999, au sommet UE d'Helsinki, il avait été prévu d'amener le contentieux devant la Cour de Justice européenne s'il n'était pas résolu par le biais de négociations avant la fin de 2004. Il est probable que cette option soit désormais caduque.

La victoire de la Nea Demokratia aux dernières élections ne remet pas en cause la bonne entente de ces dernières années dans la mesure où dans le passé, l'attitude d'un Caramanlis ou d'un Mitsotakis avait été beaucoup plus souple que celle du PASOK.

Reste le nœud gordien qu'est l'affaire chypriote. Sans entrer dans les détails, car ce n'est pas l'objet de ce texte, il faut tout de même signaler que la fin de 2003 et le début de 2004 ont été témoins de changements majeurs du côté turc, avec un élan populaire des Chypriotes turcs en faveur de la réunification, mais surtout avec les résultats des élections du 14 décembre 2003 qui ont donné un résultat partagé entre les partisans du plan Annan et ses adversaires ${ }^{82}$. Sur les 50 sièges à l'Assemblée, les deux principales formations de l'opposition - le parti républicain turc (CTP) et le Mouvement pour la paix et la démocratie (BDH) - en ont obtenu 25, tandis que les deux partis du gouvernement sortant celui de l'Unité nationale (UBP) et le Parti démocrate (OP) - en obtenaient également 25. Depuis, avec un engagement fort d'Ankara en faveur du plan 
Annan, un gouvernement a pu être fondé grâce à la participation du OP dirigé par Serdar Denktaş, fils du Président Rauf Denktaş et à l'assouplissement forcé de la position de celui-ci. Les négociations qui ont mené à un référendum auraient dû, selon le nouveau premier ministre Mehmet Ali Talat, permettre la réunification de l'île avant d'accéder ensemble à l'Union européenne le 1er mai 2004. Ce ne fut pas le cas après un "non» massif des Grecs de l'île au plan Annan malgré un «oui» du côté turc. Actuellement, les Chypriotes turcs tentent de se faire reconnaître par la communauté internationale, du moins essayent de limiter leur isolement avec l'appui de la Turquie. Cette dernière, à son tour, subit des pressions pour reconnaître la République de Chypre, désormais membre de l'Union européenne. La résolution définitive du problème chypriote sera, sans aucun doute, un catalyseur formidable pour la normalisation définitive des relations gréco-turques.

\section{BIBLIOGRAPHIE}

\section{Journaux et hebdomadaires utilisés}

Journaux turcs

Hürriyet, Cumhuriyet, Milliyet, Radikal, Finansal Forum, Alcyam, Salom

Journaux grecs

To Vima, Ta Nea, I Kathimerini

Journaux minoritaires grecs

Apoyevmatini, lho, 0 Politis

Journaux minoritaires turcs de Grèce

Gündem, Ileri, Trakya'nm Sesi

Sites Internet consultés (5 décembre 2003)

http://www.al-jazeerah.info.com/

http://www.deik.org.tr/

\section{Livres et articles cités}

Akgönül Samim, (1999), « L'émigration des musulmans de Thrace occidentale », Mésogeios, 3, 1999, p. 38-39.

Akgönül Samim, (1999), Une communauté, deux États : la minorité turco-musulmane de Thrace occidentale, Istanbul : Isis.

Akgönül Samim, (2000), « Les détentes précédentes dans les relations gréco-turques », Hellenic Studies, 8 (2), p. 107-151.

Akgönül Samim, (2001), « La détente gréco-turque et les minorités », Hellenic Studies, 9 (1), 2001, p. 49-70. 
Akgönül Samim, (2001), « Vers une nouvelle donne dans les relations gréco-turques », Les dossiers de l'IFEA, (6), p. 1-6.

Akgönül Samim, (2001), « Chypre et les minorités gréco-turques : chronique d'une prise d'otage », Gremmo-Monde arabe contemporain. Cahiers de recherches, 29 « Recherches en cours sur le problème chypriote ", p. 37-51.

Akgönül Samim, (2002), « Les activités du Patriarcat “œcuménique” du Phanar dans les années 1990 et l'opinion publique turque », CEMOTI, 33, p. 195-216.

Akgönül Samim, (2002) «Les écoles grecques de Turquie », Mésogeios, 17-18, p. 93-124.

Akgönül Samim, (2002), « Qui est Sadık Ahmet ? Le parcours d'un nationaliste turc en Grèce », Balkanologie, V, 3, p. 213-227. (http://balkanologie.revues.org/index456.html)

Akgönül Samim,(2002), "Religious Institutions of the Muslim Minority of Greece" in A Wasif Shadid, P. S Van Koningsveld (éds.), Religious Freedom and the Neutrality of the State: The position of Islam in the European Union, Leuven: Peeters, pp. 145-157.

Bilge Suat (1998), “The Phener Greek Patriarchate”, Perceptions, p. 19-34.

Çavusoglu Halim, (1993), Balkanlar'da Pomak Türkleri Tarih ve Sosyo-Kültürel Yapı, [Les Turcs Pomaks des Balkans, Histoire et vie socioculturelle], Ankara: Kôksav, 230 p.

Demir Hülva, Akar Ridvan (1994) Istanbul'un son sürgünleri, [Les derniers exils d'Istanbul ], Istanbul: Îletişim.

Denk Erdem,(1999), Egemenliği Tartışmalı Adalar: Karşılaştırmalı Bir Çalışma, [Les discussions sur les îles de l'Égée :un travail de comparaison], Ankara: Mülkiyeliler Birligi Vakfı Yayımları.

Économique Constantin, (1997), «Les îlots d'Imia dans la mer Égée : un différend créé par la force », Revue générale de Droit international public, $\mathrm{n}^{\circ}$ 101, p. 323-341.

Firat Melek, (2001), "Yunanistan'la ilişkiler”, [Les relations avec la Grèce] Oran Baskım, Türk Diş Politikası, [Politique extérieure turque], Istanbul: İletişim, tome II, p. 440-480.

Firat Melek, (1995), Une base du terrorisme en Europe : la Grèce, Ankara: Yıldız, 35 p.

Güven Erdal, (2003), Helsinki'den Kopenhag'a Kıbrıs, [Chypre, d'Helsinki à Copenhague], Istanbul: Om Yayımevi, $238 \mathrm{p}$.

Helsinki Watch Report, (1990) Destroying Ethnic Identity: the Turks of Greece, New York.

Kalivretakis Leonidas, (1996), Report on the "Limia-Imia" Islets, Athènes: Institute of Neohellenic Research Foundation.

Kuramahmut Ali (éd) (1998) Ege' de Temel Sorun: Egemenliği Tartışmalı Adalar, [La question de l'Égée : discussions sur les îles de l'Égée], Ankara :Türk Tarih Kurumu Yayımları, no. 4.

Macar Elçin, (2003), Cumhuriyet Doneminde Istanbul Rum Patrikhanesi, [Le Patriarcat rum d'Istanbul depuis le début de la République], Istanbul: Îletişim.

Macar Elçin, BenlisoyYorgo, (1997), Fener Patrikhanesi, [Le Patriarcat du Phanar], Ankara: Ayraç.

Tiliç Dogan, (1998) Utanıyorum ama gazeteciyim. Türkiye ve Yunanistan'da Gazetecilik, [Je suis embarrassé, mais je suis journaliste. Le journalisme en Turquie et en Grèce], Istanbul: Îletişim. 


\section{NOTES}

1. Melek Fuat, "Yunanistan'la ilişkiler”, Baskın Oran (dir.), Türk Diş Politikası, Istanbul, İletişim, 2001, tome II, p. 440.

2. Concernant l'attitude des journalistes populistes en Grèce et en Turquie face aux problèmes bilatéraux, voir Dogan Tiliç, Utanıyorum Ama Gazeteciyim. Türkiye ve Yunanistan'da Gazetecilik, Istanbul, İletişim, 1998, 392 p.

3. "Bayrak ya inecek, ya inecek", (Ce drapeau sera descendu ou descendu), Hürriyet, 26 janvier 1996.

4. Cumhuriyet, $1^{\mathrm{er}}$ février 1996.

5. Pour plus de détails sur le déroulement de la crise voir Melek Firat, op. cit., p. 464-468.

6. En mai 1996 une autre crise, de plus petite ampleur, mais semblable à la crise d'Imia-Kardak est survenue concernant l'îlot Gavdos/Keçi, Hürriyet, To Vima, 3 juin 1996.

7. Pour l'argumentation juridique et technique turque sur les « zones grises » voir Erdem Denk, Egemenligi Tartı şmalı Adalar: Karşılaştırmalı Bir Çalışma, Ankara. Mülkiyeliler Birligi Vakfı Yayımları, 1999, 284 p. et Ali Karamahmut, Ege 'de Temel Sorun: Egemenliği Tartışmalı Adalar, Ankara, Türk Tarih Kurumu Yayımları, $n^{\circ} 4$, 1998.- Pour l'argumentation juridique et technique grecque sur l'absence des «zones grises » voir Leonidas Kalivretakis, Report on the "Limia-Imia" Islets, Athènes, Institute of Neohellenic Research Foundation, 1996, et Constantin Economides, "Les îlots d'Imia dans la mer Égée: un différend créé par la force », Revue générale de Droit international public, $\mathrm{n}^{\circ} 101,1997$, p. 323-341.

8. Voir à ce propose un pamphlet: Fondation turque de la Démocratie, Une base du terrorisme en Europe : la Grèce, Ankara, Yıldız, 1995, 35 p.

9. Hürriyet, Milliyet, 21 octobre 1998.

10. Hürriyet, 13 novembre 1998.

11. Hürriyet, 19 novembre 1998.

12. “Italya da Def Etti” (L'Italie aussi l'a fichu dehors), Hürriyet, 17 janvier 1999.

13. "Cumhurbaşkanı Süleyman Demirel'den Yunanistan'a Sert Uyan: Atina Teror Destekçisi” (Mise en garde sévère du Président Demirel à la Grèce: Athènes soutient le terrorisme), Cumhuriyet, 23 février 1999.

14. Ta Néa, $1^{\mathrm{er}}$ mars 1999.

15. "Yorgo Papandreu- Ismail Cem Görüşmesi Bugün Ankara'da Gerçekleşiyor” (La rencontre entre Giorgos Papandréou et Ismail Cey aura lieu aujourd'hui à Ankara), Cumhuriyet, 20 janvier 2000.

16. Près de 20000 morts, 40000 blessés.

17. "Efharisto Poli File" (Merci beaucoup, ami), Hürriyet, 21 août 1999.

18. Le journal télévisé d'ATV, 22 août 1999.

19. Les déclarations du ministre de la Santé Osman Durmuş. Cumhuriyet, 26 août 1999.

20. Cumhuriyet, 9 septembre 1999.

21. Cumhuriyet, 7 août 1990.

22. Halim Çavuşoglu, Batı Trakya Türkleri'nin Yasak Bölge'de bir Yerleşim Birimi. Pomaklar'la Meşkam Şahin Nahiyesinin Sosyo-kültürel Yapısı, Ankara, Thèse de Doctorat, Université de Hacettepe, 1991, p. 34. (Cette thèse a donné lieu à une publication restreinte : Halim Çavuşoglu, Balkanlar'da Pomak Türkleri- Tarih ve Sosyo-Kültürel Yapl, Ankara, Köksav, 1993.)

23. Il est utile de préciser qu'en Bulgarie également la frontière gréco-bulgare fut une zone militaire interdite pendant toute la guerre froide.

24. Samim Akgönül, «L'émigration des musulmans de Thrace occidentale » Mésogeios, 3, 1999, p. 38-39.

25. Helsinki Watch Report, Destroying Ethnic Identity: the Turks of Greece, New York, 1990, p. 11.

26. PE 202. 357/fin., 27 janvier 1993.

27. Voir par exemple la liste publiée dans Gündem, 6 février 2001. 
28. Pour une analyse de ces «tracasseries » voir Samim Akgönül, Une communauté, deux États : la minorité turco-musulmane de Thrace occidentale, Istanbul, Isis, 1999, p. 131-155.

29. Pour l'implication des relations bilatérales sur les minorités voir Samim Akgönül, «La détente gréco-turque et les minorités », Hellenic Studies, 9 (1), 2001, p. $49-70$ et Samim Akgönül, "Chypre et les minorités gréco-turques: chronique d'une prise d'otage », Gremmo-Monde arabe contemporain. Cahiers de recherches, 29 "Recherches en cours sur le problème chypriote », 2001, p. 37-51. 30. Pour une analyse détaillée des activités du Patriarcat voir Elçin Macar, Cumhuriyet Doneminde Ïstanbul Rum Patrikhanesi, Istanbul, iletişim, 2003, p. 239 et passim et Samim Akgönül, «Les activités du Patriarcat “œcuménique" de Phanar dans les années 1990 et l'opinion publique turque », CEMOTI, 33, 2002, p. 195-216.

31. Hülya Demir, Rıdvan Akar, Istanbul 'un son sürgünleri, Istanbul, Iletişim, 1994, p. 162.

32. Voir par exemple Apoyevmatini, 6 août 1974, 7 août 1974.

33. Salom, $1^{\text {er }}$ novembre 2002.

34. Les efforts soudains de la Grèce pour soutenir la Turquie dans le processus d'adhésion, preuve d'un changement d'attitude radical, en surprend plus d'un à Ankara et sont parfois accueillis avec suspicion, "Yunanistan Türkiye'nin Avrupa Birligi Konusunda Hukuk Danışmanı olacak” (La Grèce sera le conseiller juridique de la Turquie à propos de l'Union européenne), Cumhuriyet, 29 décembre 1999.

35. Ta Néa, 21 mai 2003.

36. "Patrik Bartholomeos : Atina'ya Cami Yaptımalı" (Le Patriarche Vartholoméos: il faut construire une mosquée à Athènes), Milliyet, $1^{\mathrm{er}}$ août 2000.

37. “Türk-Yunan Dostluk Günleri: Türk ve Yunan Ogrencilerin ilk Buluşması" (Les journées grécoturques d'amitié : première rencontre des étudiants grecs et turcs), Milliyet, 25 octobre 1999.

38. "Tarih Yeniden Yazıliyor" (On réécrit l'Histoire), Cumhuriyet, 27 octobre 1999, "Ders Kitapları Ayıklanmalı" (Les livres d'Histoire doivent être nettoyés), Milliyet, 21 octobre 1999.

39. Akşam, 16 mai 2003.

40. Il existe un Conseil d'affaires gréco-turc très actif au sein de Diş Ekonomik ilişkiler Kurulu (Conseil des Relations économiques extérieures) : http://www.deik.org.tr/

41. "Türk-Yunan İş Seferleri Başlıyor" (Les rencontres d'affaires gréco-turques commencent) Finansal Forum, 31 août 1999.

42. "Dostluk Treninin Yunanistan Turu Iki Ulusun Barış Özleminin Kanıtı Oldu" (Le circuit en Grèce du train d'amitié a été la preuve que les deux nations désirent la paix), Milliyet, 16 novembre 1999.

43. “Atina'da Dostluk Rüzgâr” (Vent d'amitié à Athènes), Radikal, 21 avril 2000.

44. Je crois que la question chypriote n'est pas la cause des mauvaises relations gréco-turques, mais en est la principale conséquence. Les causes me semblent plus profondes et complexes, j'ai tenté de les analyser dans Samim Akgönül, «Vers une nouvelle donne dans les relations grécoturques ", Les dossiers de l'IFEA, (6), 2001, p. 1-46.

45. “Atina işleri Brüksel'e Havale Etti” (Athènes a transféré toutes les affaires à Bruxelles), Cumhuriyet, 5 janvier 2000.

46. Melek Firat, op. cit., p. 454.

47. “Denktaş Helsinki Sonuçlarından Rahatsız: 'Kıbrıs Türkü Rehin Kaldı”' (Dentaş n'est pas content d'Helsinki : les Turcs de Chypre sont devenus des otages), Cumhuriyet, 14 décembre 1999.

48. "Dışişleri Bakanı Cem, AB ile Kıbrıs'ın Karıştırılmaması Gerektiğini Soyledi” (Le ministre des Affaires étrangères Cem a déclaré qu'il ne fallait pas mélanger l'Union européenne et Chypre), Cumhuriyet, 14 décembre 1999.

49. En décembre 2002 et janvier 2003, Nicosie a été la scène de manifestations populaires sans précédent où des milliers de Chypriotes turcs ont montré leur désaccord avec la politique de Denktaş. 
50. Cumhuriyet, 15 janvier 2003.

51. Pour une description chronologique des événements chypriotes des quatre dernières années, voir Erdal Güven, Helsinki'den Kopenhag'a Kıbrıs, Istanbul, Om Yayınevi, 2003.

52. Suivant ce libre passage au sud de 1'île, 20000 Chypriotes turcs ont fait la demande d'un passeport de la République de Chypre pour profiter de la libre circulation européenne lors de l'intégration, totale de 1'île à l'UE. "Rum Pasaportuna 20 bin başvuru" (20 mille demandes pour le passeport chypriote grec).

53. Ta Néa, 12 décembre 1999.

54. Inal Batu, "Yunanistan Açıklamalarında Samimi Değil" (La Grèce n'est pas sincère), Milliyet, 20 avril 1997.

55. Cumhuriyet, 25 mai 2003.

56. Sema Kalaycınğlu, "Türk Yunan Dostluguna Gastronomik Bir Yaklaşım: Kokoreç Diplomasisi” (Une approche gastronomique à l'amitié gréco-turque: la diplomatie de Kokoreç), Finansal Forum, 27 janvier 2000.

57. “Ege'de Balıkçının Aşkı evlilikle bitecek" (Dans la mer Égée, l'amour du pêcheur se terminera avec un mariage), Milliyet, 14 janvier 2000.

58. Pour la personnalité et la carrière fulgurante de ce personnage voir Samim Akgônül, « Qui est Sadık Ahmet ? Le parcours d'un nationaliste turc en Grèce ", Balkanologie, V, 3, décembre 2002, p. 213-227. (http://balkanologie.revues.org/index456.html)

59. Trakya' nın Sesi, 10 juin 1985. Ileri, 8 juin 1985.

60. Ileri, 14 juin 1985.

61. Trakya'nin Sesi, 31 décembre 1985.

62. Pour plus de détails sur l'affaire des muftis, voir Samim Akgönül, "Religious Institutions of the Muslim Minority of Greece" in A. Wasif Shadid, P. S. van Koningsveld (éds. ), Religious Freedom and the Neutrality of the State: The position of Islam in the European Union, Leuven, Peeters, 2002, pp. 145-157.

63. Pour une vue d'ensemble des problèmes scolaires des écoles grecques en Turquie, voir Samim Akgonül, «Les écoles grecques de Turquie », Mésogeios, (17-18), 2002, p. 93-124.

64. Heybeliada, une des îles de Prince, au large d'Istanbul dans la Mer de Marmara, qui abrite, comme les autres d'ailleurs, une importante communauté de minoritaires.

65. Düstur, 5. Tertip, t. 10, 1971, p. 1176-1229. 12 janvier 1971.

66. Elçin Macar, Yorgo Benlisoy, Fener Patrikhanesi, Ankara, Ayraç, 1997, p. 67.

67. Suat Bilge, "The Phener Greek Patriarchate", Perceptions, 1998, p. 27.

68. Apoyevmatini, 26 septembre 1999, ou lorsqu'elle s'est adressée aux orthodoxes américains en mai 2000, Cumhuriyet, 18 mai 2000.

69. Milliyet, 21 octobre 1999.

70. Iho, 3 février 1992.

71. Tavşanoğlu Leyla, "Heybeliada Ruhban Okulu Türkiye'nin önünü açar" (L'école théologique de Halki peut lever les obstacles devant la Turquie), Cumhuriyet, 19 octobre 1999.

72. Cumhuriyet, 19 octobre 1999.

73. Apoyevmatirni, 2 novembre 1998.

74. Apoyevmatini, 8 novembre 1998.

75. 0 Politis, décembre 1998.

76. http://www.mfa.gov.tr/default.tr.mfa déclaration de presse $n^{\circ} 85-12,12$ novembre 1998, la déclaration réserve une grande partie au traitement des fondations pieuses des Turcs de Grèce par le gouvernement grec.

77. Voir sur cette question Samim Akgönül, «Les détentes précédentes dans les relations grécoturques », Hellenic Studies, 8 (2) 2000, p. 107-151.

78. Liter Türkmen, “Türkiye, Yunanistan ve Kıbrıs” in Hürriyet, 22 janvier 2004.

79. Ta Néa, 26 janvier 2004. 
80. Gündem, 26 janvier 2004.

81. “Bartolomeos'tan Castro'ya teşekkür”, Milliyet, 27 janvier 2004.

82. "Élections à Chypre nord: le gouvernement a perdu, l'opposition n'a pas gagné », AFP, 15 décembre 2003.

\section{RÉSUMÉS}

La fin $\mathrm{du} \mathrm{xx}^{\mathrm{e}}$ siècle et le début $\mathrm{du} \mathrm{XxI} \mathrm{e}^{\mathrm{e}}$ siècle ont été porteurs de changements dans les relations gréco-turques pour des raisons à la fois globales et locales. La fin du monde bipolaire, le poids de plus en plus important de l'Union européenne dans le sud-est européen, mais aussi une mutation des visions dans les relations bilatérales ont fait que globalement ces relations se sont améliorées. Ces améliorations ont pris parfois des formes étonnement brutales comme au lendemain du tremblement de terre en Turquie en 1999. Mais à côté de ces ruptures aussi soudaines que rares, il y a eu, comme dans l'ensemble de l'histoire de relations bilatérales, des évolutions sensibles, surtout en ce qui concerne la situation des minorités réciproques. Et enfin, en dépit de cette amélioration, il subsiste toujours certains domaines du conflit gréco-turc qui connaissent une stagnation comme celui des différends liés à la mer Égée.

The end of the 20th century and the beginning of the 21th century brought changes in the Greek Turkish relations for global and local reasons. The end of the bipolar world, the increasingly important weight of the European Union in European South-East, but also a change of vision in the bilateral relations improved these relations. These improvements took forms sometimes brutal as on the following day of the earthquake in Turkey in 1999. However, even if these ruptures are as sudden as rare, there were too, as in the whole of the history of bilateral relations, significant slow evolutions, especially with regard to the situation of the reciprocal minorities had been observed. And finally, in spite of this improvement, there are always certain fields of the Greek-Turkish conflict that show a stagnation as the disagreements relative to the Aegean Sea.

XX. yüzyıl sonları ve XXI. yüzyıl başları, küresel ve yerel sebeplerden dolayı Türk-Yunan ilişkilerinde değişimlerin görüldüğü dönemlerdir. Soğuk savaşın bitmesi, Güneydoğu Avrupa'da günden güne önem kazanan Avrupa Birliği'nin varlı̆̆ı ve aynı zamanda ikili ilişkilerdeki vizyon değişimi bu ilişkilerin iyileşmesinde genel anlamda etken olmuştur. Bu ilişkilerin iyiye gitmesi kimi zaman şaşırtıcı ve şiddetli bir şekilde kendini göstermiştir, 1999 Türkiye depreminde olduğu gibi. Ancak ani olduğu kadar nadir de olan bu kırılmaların yanında, tarihte tüm ikili ilişkilerde olduğu gibi, belirgin dönüşümler de olmuştur, özellikle azınlıkların karşılıklı durumuyla ilgili. Son olarak, bu iyileşmeye rağmen, hâlâ Türk-Yunan sorunun durgunluk gösteren bazı alanlarının mevcut olduğunu söyleyebiliriz, Ege sorunu gibi. 


\section{INDEX}

Index géographique : Grèce, Turquie, Chypre

Mots-clés : relations gréco-turques, Patriarcat, Phanar, Phanar, question chypriote, minorités

Keywords : Greek-Turkish relations, Patriarchate, Phener, Cyprus question, Minorities, Greece, Turkey, Contemporary history, History

motsclestr Türk-Yunan ilişkileri, Patrikhane, Fener, Kıbrıs sorunu, Ege sorunu, Azınlıkları sorunu, Yunanistan, Türkiye, Kıbrıs, Yirminci Yüzyıl, Çağdaş tarihin, Tarih, Uluslararası ilişkiler

Thèmes : Histoire, Relations internationales

motsclesmk ГРЧКО-ТУРСКИ ОДНОСИ, ПАТРИЈАРХАТ, ФАНАР, КИПАРСКОТО ПРАШАҢЕ, ПРОБЛЕМОТ СО ЕГЕЈСКОТО МОРЕ, МАЛЦИНСКИ ПРОБЛЕМИ, ГРЦИЈА, ТУРЦИЈА, КИПАР, ДВАЕСЕТТИОТ ВЕК, СОВРЕМЕНАТА ИСТОРИЈА, ИСТОРИЈА, МЕЃУНАРОДНИ ОДНОСИ

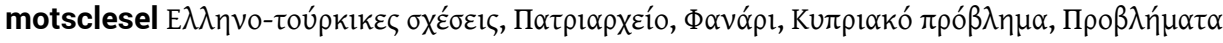

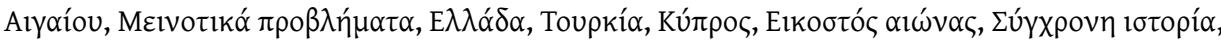

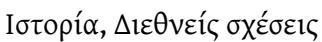

Index chronologique : vingtième siècle 